\title{
Higgs dark matter from a warped extra dimension the truncated-inert-doublet model
}

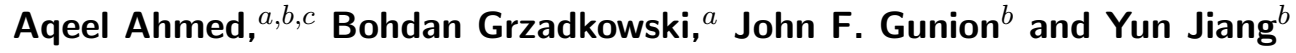 \\ ${ }^{a}$ Faculty of Physics, University of Warsaw, \\ Pasteura 5, 02-093 Warsaw, Poland \\ ${ }^{b}$ Department of Physics, University of California, \\ Davis, CA 95616, U.S.A. \\ ${ }^{c}$ National Centre for Physics, Quaid-i-Azam University Campus, \\ Shahdra Valley Road, Islamabad 45320, Pakistan \\ E-mail: aqeel.ahmed@fuw.edu.pl, bohdan.grzadkowski@fuw.edu.pl, \\ jfgunion@ucdavis.edu, yunjiang@ucdavis.edu
}

ABSTRACT: We construct a 5D $\mathbb{Z}_{2}$-symmetric model with three D3-branes: two IR ones with negative tension located at the ends of an extra-dimensional interval and a UVbrane with positive tension placed in the middle of the interval - IR-UV-IR model. The background solutions for this geometric setup are found without and with taking into account the backreaction of the matter fields. A 5D SU(2) Higgs doublet is employed as the Goldberger-Wise stabilizing field in this geometry and solutions of the 5D coupled scalar-gravity equations are found by using the superpotential method. Within this setup we investigate the low-energy (zero-mode) effective theory for the bulk Standard Model (SM) bosonic sector. The $\mathbb{Z}_{2}$-even zero-modes correspond to known standard degrees of freedom, whereas the $\mathbb{Z}_{2}$-odd zero modes might serve as a dark sector. The effective lowenergy scalar sector contains a scalar which mimics the SM Higgs boson and a second stable scalar particle (dark-Higgs) is a dark matter candidate; the latter is a component of the zero-mode of the $\mathbb{Z}_{2}$-odd Higgs doublet. The model that results from the $\mathbb{Z}_{2}$-symmetric background geometry resembles the Inert Two Higgs Doublet Model. The effective theory turns out to have an extra residual $\mathrm{SU}(2) \times \mathrm{U}(1)$ global symmetry that is reminiscent of an underlying 5D gauge transformation for the odd degrees of freedom. At tree level the SM Higgs and the dark-Higgs have the same mass; however, when leading radiative corrections are taken into account the dark-Higgs turns out to be heavier than the SM Higgs. Implications for dark matter are discussed; it is found that the dark-Higgs can provide only a small fraction of the observed dark matter abundance.

Keywords: Phenomenology of Large extra dimensions, Phenomenology of Field Theories in Higher Dimensions

ARXIV EPRINT: 1504.03706 


\section{Contents}

1 Introduction 1

$2 \quad \mathrm{~A} \mathbb{Z}_{2}$ symmetric warped extra-dimension and KK-parity 5

2.1 The IR-UV-IR model: without backreaction 5

$\begin{array}{lll}2.2 & \text { The IR-UV-IR model: with backreaction } & 7\end{array}$

2.3 Warped KK-parity 11

3 SM EWSB by bulk Higgs doublet - the truncated-inert-doublet model 11

$\begin{array}{ll}3.1 \text { Quantum corrections to scalar masses } & 18\end{array}$

$\begin{array}{lll}3.2 & \text { Dark matter relic abundance } & 21\end{array}$

$\begin{array}{lll}4 & \text { Summary } & 23\end{array}$

A SSB in the IR-UV-IR model: the Abelian Higgs mechanism 25

A.1 SSB by vacuum expectation values of KK modes 26

A.2 SSB by a vacuum expectation value of the 5D Higgs field 30

\section{Introduction}

The seminal work of Randall and Sundrum (RS) [1] provides an elegant solution to the hierarchy problem. Their proposal involves an extra-dimension with a non-trivial warp factor due to the assumed anti-de Sitter (AdS) geometry along the extra-dimension. In their model an AdS geometry on an $S_{1} / \mathbb{Z}_{2}$ orbifold is considered which is equivalent to a line-element $0 \leq y \leq L$, where $y$ is the coordinate of the fifth-dimension and $L=\pi r_{c}$, with $r_{c}$ being the radius of the circle in the fifth-dimension. Moreover, their model involves two D3-branes localized on the fixed points of the orbifold, a "UV-brane" at $y=0$ and an "IR-brane" at $y=L$ (our nomenclature will become clear below), see figure 1 . The solution for the RS geometry is $[1,2]$,

$$
d s^{2}=e^{2 A(y)} \eta_{\mu \nu} d x^{\mu} d x^{\nu}+d y^{2}, \quad \text { with } \quad A(y)=-k|y|,
$$

where $k$ is the inverse of the AdS radius. In the original RS1 model [1] it was assumed that the Standard Model (SM) is localized on the IR-brane, whereas gravity is localized on the UV-brane and propagates through the bulk to the IR-brane. They famously showed that if the 5D fundamental theory involves only one mass scale $M_{*}$ - the Planck mass in 5D - then, due to the presence of non-trivial warping along the extra-dimension, the effective mass scale on the IR-brane is rescaled to $m_{K K} \sim k e^{-k L} \sim \mathcal{O}(\mathrm{TeV})$ and hence ameliorates the hierarchy problem for mild values of $k L \sim \mathcal{O}(35)$. 


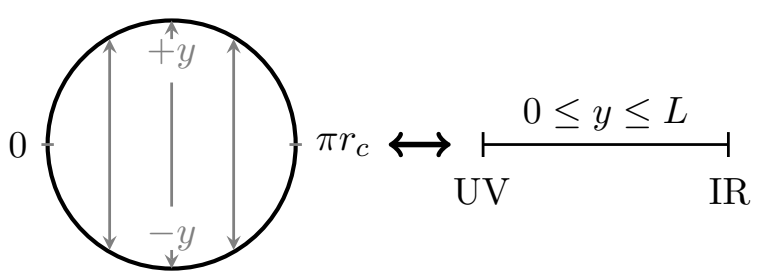

Figure 1. Cartoon of RS1 geometry.

Soon after the RS proposal, many important improvements to the model were considered. First, a stabilization mechanism for the RS1 setup was proposed by Goldberger and Wise [3]; it employs a real scalar field in the bulk of AdS geometry with localized potentials on both of the branes, see also [4]. A second interesting observation, which could potentially solve the fermion mass hierarchy problem within the SM, was made by many groups [5-9]. The core idea of these works was to allow all the SM fields to propagate in the RS1 bulk, except the Higgs field which was kept localized on the IR-brane. In this way, the zero-modes of these bulk fields correspond to the SM fields and the overlap of $y$-dependent profiles of fermionic fields with the Higgs field could generate the required fermion mass hierarchy. To suppress the electroweak (EW) precision observables, the symmetry of the gauge group was enhanced by introducing custodial symmetry in ref. [10]. The common lore, in the RS1 model and its extensions, was to keep the Higgs field localized on the IRbrane in order to solve the hierarchy problem. The first attempt to consider the Higgs field in the bulk of RS1 was made by Luty and Okui [11]. They employed AdS/CFT duality ${ }^{1}$ to argue that a bulk Higgs scenario can address the hierarchy problem by making the Higgs mass operator marginal in the dual CFT.

A study of electroweak symmetry breaking (EWSB) within the bulk Higgs scenario was first performed in the RS1 setup by Davoudiasl et al. [14]; they showed that the zeromode of the bulk Higgs is tachyonic and hence could lead to a vacuum expectation value (vev) at the $\mathrm{TeV}$ scale. Recently there have been many studies where a bulk Higgs scenario is considered from different perspectives - see for example: a study with custodial symmetry in the Higgs sector [15]; models with a soft wall setup [16]; bulk Higgs mediated FCNC's [17]; suppression of EW precision observables by modifying the warped metric near the IR-brane [18-20]; and, a bulk Higgs as the modulus stabilization field (Higgs-radion unification) [21]. Different phenomenological aspects after the Higgs discovery were explored in [22-29]. These phenomenological studies show that the RS1 model with bulk SM fields and its descendants with modified geometry (RS-like warped geometries in general) are consistent with the current experimental bounds and EW precision data.

A separate category of generalization of the RS models is based on the assumption that the singular branes are replaced with thick branes which are smooth field configurations of the bulk scalar field, see e.g. [4] and [30, 31].

As we discussed above, RS-like warped geometries, being consistent with the experimental data, offer an attractive solution to many of the fundamental puzzles of the SM, mostly through geometric means. In the same spirit, one can ask if RS-like warped extra-

\footnotetext{
${ }^{1}$ For the phenomenological applications of AdS/CFT with RS1 geometry, see for example [12, 13].
} 
dimensions can shed some light on another outstanding puzzle of SM, the lack of a candidate for dark matter (DM) which constitutes $83 \%$ of the observed matter in the universe [32]. It appears that unlike (flat) universal extra-dimensions (UED), where the KK-modes of the bulk fields can be even and odd under KK-parity (implying that the lowest KK-odd particle (LKP) could be a natural dark matter candidate [33, 34]), RS1-like models (involving two branes and warped bulk) are unable to offer an analogue of KK-parity. The reason lies in the fact that the RS1 geometry is just a single slice of AdS space and, since warped, cannot be symmetric around any point along the extra-dimension and hence does not allow a $K K$-parity. As a result it cannot accommodate a realistic dark matter candidate. To cure this problem in the warped geometries, usually extra discrete symmetries are introduced such that the SM fields are even while the DM is odd under such discrete symmetries in order to make it stable [35-38]. Another way to mend this problem in warped geometries is to introduce an additional hidden sector with some local gauge symmetries such that only DM is charged under the hidden sector gauge symmetries and it couples to the SM very weakly [39, 40], (see also [41]).

An alternative to introducing additional symmetries, is to extend the RS1-like warped geometry in such a way that the whole geometric setup becomes symmetric around a fixed point in the bulk. Two $\mathbb{Z}_{2}$ symmetric warped configurations are possible. In the first, two identical AdS patches are symmetrically glued together at a UV fixed point, while in the second two identical AdS pathes are symmetrically glued together at an IR fixed point. The geometric configuration when the two AdS copies are glued together at the UV fixed point will be referred as "IR-UV-IR geometry", whereas the geometry corresponding to the setup when two AdS copies are glued at the IR fixed point is called "UV-IR-UV geometry". We will only consider the IR-UV-IR geometric setup - it is straight forward to extend our analysis to the UV-IR-UV geometries. (A common pathology associated with this latter type of geometry is the appearance of ghosts.) We consider an interval $y \in[-L, L]$ in the extra-dimension, where on each end of the interval $y= \pm L$ there is a D3 brane with negative tension (in section 2.1 it will be clear why we need negative tension branes) and at the center of the interval, $y=0$, we place a positive tension brane where we assume that gravity is localized. ${ }^{2}$ We call the boundary branes "IR-branes" and the brane at $y=0$ we term the "UV-brane". The IR-UV-IR geometry and a pictorial description of such a geometric setup is shown in figure 2. Since the brane tensions of the two IR-branes are the same, this geometry is $\mathbb{Z}_{2}$ symmetric. We are aware of only two earlier attempts to construct a similar setup. The first [42] treated the lowest odd KK gauge mode as the DM candidate. The second employed a kink-like UV thick brane [43] and the corresponding dark-matter was the first odd KK-radion [44].

In this work, we place all the SM fields, including the Higgs doublet, in the bulk of the IR-UV-IR geometry. We calculate the background solutions for our geometric setup without and with taking into account the backreaction of matter fields. Since only 5D Higgs doublet, present in the bulk as well on the branes, acquires $y$-dependent vev, therefore we

\footnotetext{
${ }^{2}$ One can smooth the singular branes in our setup by appropriate scalar field configurations - for smooth brane modeling see for example [30] and references therein.
} 


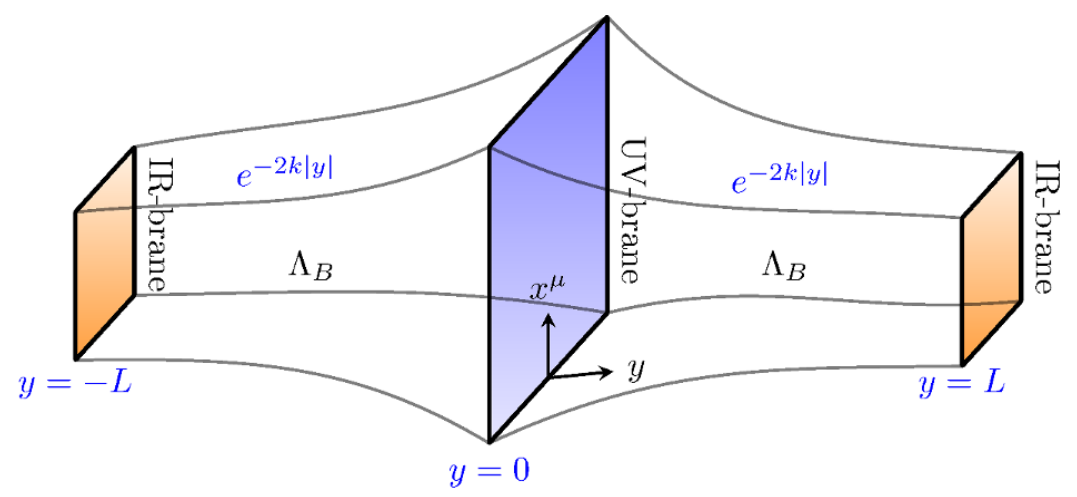

Figure 2. The geometric configuration for IR-UV-IR setup, the parameters are defined in section 2.1 .

solve the full 5D scalar-gravity coupled set of Einstein equations to get solutions which address the gauge hierarchy problem. Here 5D SU(2) Higgs doublet plays the role of the Goldberger-Wise stabilization field and the values of Higgs vevs at the UV- and IR-brane fix the distance between the branes. We find that for a weak backreaction, the UV-brane Higgs vev has to be much smaller than that of the IR-brane. Moreover we show that in order to have $4 \mathrm{D}$ cosmological constant zero at the IR branes one needs precisely one fine-tuning, similar to RS1 [3, 4].

The geometric $\mathbb{Z}_{2}$ parity ( $y \rightarrow-y$ symmetry) leads to "warped KK-parity", i.e. there are towers of even and odd KK-modes corresponding to each bulk field. In the weak backreaction scenario we focus on EWSB induced by the bulk Higgs doublet and low energy aspects of the $4 \mathrm{D}$ effective theory for the even and odd zero-modes assuming the KK-mass scale is high enough $\sim \mathcal{O}$ (few) $\mathrm{TeV}$. In the effective theory the even and odd Higgs doublets mimic a two-Higgs-doublet model (2HDM) scenario - the truncated inert-doublet model - with the odd doublet similar to the inert doublet but without corresponding pseudoscalar and charged scalars. All the parameters of this truncated 2HDM are determined by the fundamental 5D parameters of the theory and the choice of boundary conditions (b.c.) for the fields at $y= \pm L$. (Note that the boundary or "jump" conditions at $y=0$ follow from the bulk equations of motion in the case of even modes, whereas odd modes are required to be zero by symmetry.) There are many possible alternative choices for the b.c. at $\pm L$. We allow the $y$-derivative of a field to have an arbitrary value at $\pm L$ as opposed to requiring that the field value itself be zero, i.e. we employ Neumann or mixed b.c. rather than Dirichlet b.c. at $\pm L$. Only the former yields a non-trivial theory allowing spontaneous symmetry breaking (SSB), whereas the latter leads to an explicit symmetry breaking scenario in which there are no Goldstone modes and the gauge bosons do not acquire mass. With these choices, the symmetric setup yields an odd Higgs zero-mode that is a natural candidate for dark matter. We compute the one-loop quadratic (in cutoff) corrections to the two scalar zero modes within the effective theory and discuss their mass splitting. The dark matter candidate is a WIMP - we calculate its relic abundance in the cold dark matter paradigm. 
The paper is composed as follows. In section 2, we setup the IR-UV-IR geometric configuration and provide background solutions without and with backreaction due to the presence of matter contents in the bulk and on the branes. We also discuss the manifestation of KK-parity due the $\mathbb{Z}_{2}$ geometric setup. Section 3 contains the main part of our work. There, we focus on EWSB for the SM gauge sector due to the bulk Higgs doublet in our $\mathbb{Z}_{2}$ symmetric geometry and obtain a low-energy $4 \mathrm{D}$ effective theory containing all the SM fields plus a real scalar — a dark matter candidate - which is odd under the discrete $\mathbb{Z}_{2}$ symmetry. In the subsequent two subsections of section 3 , we consider the quantum corrections to the scalar masses below the KK-scale $\sim \mathcal{O}$ (few) $\mathrm{TeV}$ and explore the possible implications of the dark-matter candidate by calculating its relic abundance. We summarize and give our conclusions in section 4 . We supplement the main text with an Abelian Higgs mechanism, with a complex scalar field and a gauge field, in our background geometry in appendix A. In the Abelian case we lay down the foundation for SSB due to bulk Higgs, which is useful in the main text for the case of EWSB of the SM. Two apparently different approaches are considered to study SSB in the Abelian case: ( $i$ ) SSB by vacuum expectation values of the KK modes; and, (ii) SSB via a vacuum expectation value of the 5D Higgs field. Low energy (zero-mode) 4D effective theories are obtained within the two approaches and we find that the effective theories are identical up to corrections of order $\mathcal{O}\left(m_{0}^{2} / m_{K K}^{2}\right)$, where $m_{0}$ and $m_{K K}$ are the zero-mode mass and KK-mass scale, respectively.

\section{$2 \quad \mathrm{~A} \mathbb{Z}_{2}$ symmetric warped extra-dimension and KK-parity}

In this section we provide the background solution for the $\mathbb{Z}_{2}$ symmetric background (IRUV-IR) geometry without and with backreaction due to the 5D matter fields and show how KK-parity is manifested within this warped geometric setup.

\subsection{The IR-UV-IR model: without backreaction}

We consider the IR-UV-IR warped geometry compactified on an interval, $-L \leq y \leq L$, where a UV-brane with positive tension is located at $y=0$, and two negative tension IR-branes are located at $y= \pm L$. Note that the end points of the interval at $y= \pm L$ are not the fixed points of the $\mathbb{Z}_{2}$, the only fixed point is at $y=0$, which is different from the $S_{1} / \mathbb{Z}_{2}$ orbifold (RS1 geometry) where $y=0$ and $y=L$ are both fixed points of the $\mathbb{Z}_{2}$. The 5D gravity action for such a geometry, without taking into account any backreaction due to the presence of matter content, can be written as, ${ }^{3}$

$$
S_{G}=\int d^{5} x \sqrt{-g}\left\{\frac{R}{2}-\Lambda_{B}-\lambda_{\mathrm{UV}} \delta(y)-\lambda_{\mathrm{IR}} \delta(y+L)-\lambda_{\mathrm{IR}} \delta(y-L)\right\}+S_{G H},
$$

where $R$ is the Ricci scalar, $\Lambda_{B}$ is the bulk cosmological constant and $\lambda_{\mathrm{UV}}\left(\lambda_{\mathrm{IR}}\right)$ are the brane tensions at the UV(IR) fixed points. Above and henceforth the Dirac delta functions at $y= \pm L$ are defined in such a way that their integral is $1 / 2$. Since our geometry is

\footnotetext{
${ }^{3}$ We use the metric signature $(-,+,+,+,+)$ and the unit system such that the 5 D Planck mass $M_{*}=1$.
} 
compact with boundaries, the action contains the Gibbons-Hawking boundary term, ${ }^{4}$

$$
S_{G H}=-\int_{\partial \mathcal{M}} d^{4} x \sqrt{-\hat{g}} \mathcal{K}
$$

where $\mathcal{K}$ is the intrinsic curvature of the surface of the boundary manifold $\partial \mathcal{M}$, given by

$$
\mathcal{K}=-\hat{g}^{\mu \nu} \nabla_{\mu} n_{\nu}=\hat{g}^{\mu \nu} \Gamma_{\mu \nu}^{M} n_{M},
$$

with $n_{M}$ being the unit normal vector to the surface of the boundary manifold $\partial \mathcal{M}$ and $\hat{g}_{\mu \nu}$ is the induced boundary metric. For the $5 \mathrm{D}$ manifold with $4 \mathrm{D}$ Poincaré invariance $\left(n^{5}=1\right.$ and $n^{\mu}=0$ ), the intrinsic curvature reduces to

$$
\mathcal{K}=-\frac{1}{2} \hat{g}^{\mu \nu} \partial_{5} \hat{g}_{\mu \nu}
$$

The solution of the Einstein equations resulting from the above action is the RS metric (1.1), where the AdS curvature $k$ is related to $\Lambda_{B}$ by

$$
\Lambda_{B}=-6 k^{2} .
$$

Since the above setup is compactified on an interval $y \in[-L, L]$, rather than on a circle as in RS1, one needs to be careful and show that the solution (1.1) is compatible with the boundaries and that the effective $4 \mathrm{D}$ cosmological constant is zero, see also [45]. We will see below that we need a fine tuning between the $5 \mathrm{D}$ cosmological constant $\Lambda_{B}$ and the brane tensions $\lambda_{\mathrm{UV}, \mathrm{IR}}$ in order to get zero $4 \mathrm{D}$ cosmological constant. One can calculate the effective $4 \mathrm{D}$ cosmological constant $\Lambda_{4}$ from the action (2.1) by integrating out the extra-dimension,

$$
\Lambda_{4}=-\int_{-L}^{L} d y \sqrt{-g}\left\{\frac{R}{2}-\Lambda_{B}-\lambda_{\mathrm{UV}} \delta(y)-\lambda_{\mathrm{IR}}[\delta(y+L)+\delta(y-L)]\right\}+\left.\sqrt{-\hat{g}} \mathcal{K}\right|_{-L} ^{L},
$$

where $R=-20 A^{\prime 2}-8 A^{\prime \prime}$ and $\Lambda_{B}=-6 A^{\prime 2}$ corresponding to the solution (1.1). Using $A(y)=-k|y|$ we find,

$$
\Lambda_{4}=\left(\lambda_{\mathrm{UV}}-6 k\right)+\left(\lambda_{\mathrm{IR}}+6 k\right) e^{-4 k L},
$$

which can only be zero if

$$
\lambda_{\mathrm{UV}}=-\lambda_{\mathrm{IR}}=6 k .
$$

This result explicitly shows that one needs a positive tension brane at $y=0$ and two negative tension branes at $y= \pm L$ in order to obtain zero $4 \mathrm{D}$ cosmological constant. This is the usual fine tuning which appears in brane world scenarios $[1,3,4]$. Hence we have a 5D geometry with AdS solution (1.1) with negative bulk cosmological constant and a positive tension brane in the middle and two equal negative tension branes at the end of the interval, see figure 2 .

We would like to mention here that we are considering a rigid IR-UV-IR geometry where the distance $L$ is tuned in order to solve the hierarchy problem. To stabilize the

\footnotetext{
${ }^{4}$ The Gibbons-Hawking boundary term is needed in order to cancel variation of the Ricci scalar at the boundaries so that the RS metric (1.1) is indeed a solution of the Einstein equations of motion.
} 
IR-UV-IR setup one may consider a mechanism like the one proposed by Goldberger and Wise (GW) [3, 4] introducing a bulk scalar field with appropriate brane potentials such that the energy minimization would set the size of the $5 \mathrm{D}$ interval and yields a compactification scale that would solve the hierarchy problem. Since one of our aims is to analyze EWSB due to a $5 \mathrm{D} \mathrm{SU}(2)$ Higgs doublet in the IR-UV-IR model, therefore it is natural to consider the bulk SU(2) Higgs doublet as the GW stabilizing field. We explore this option in the following subsection. A similar analysis for the case of RS1 has been considered in ref. [21]. However, there the full scalar-gravity coupled equations are not solved, the authors adopt a small backreaction anstaz. In this work, we are using so-called superpotential method to solve the full scalar-gravity coupled equations analytically.

\subsection{The IR-UV-IR model: with backreaction}

In this subsection we employ an SU(2) Higgs doublet in the bulk of IR-UV-IR model and obtain the background solutions for the 5D scalar-gravity coupled theory. Although our solution generating technique is adopted for a specific geometric configuration (IR-UV-IR model), this approach can be used to solve the Higgs-gravity backreaction in any warped extra-dimensional model (e.g. RS1) with a bulk Higgs. We use the following most general 4D Poincaré invariant metric ansatz:

$$
d s^{2}=e^{2 A(y)} \eta_{\mu \nu} d x^{\mu} d x^{\nu}+d y^{2},
$$

where $A(y)$ is a general $y$-dependent warp-function and $\eta_{\mu \nu}$ represents the $4 \mathrm{D}$ Minkowski metric. We consider the following scalar-gravity action for our model,

$$
\begin{aligned}
S_{S G}=\int d^{5} x \sqrt{-g} & \left\{\frac{R}{2}-\left|D_{M} H\right|^{2}-V_{B}(H)\right. \\
& \left.-V_{\mathrm{UV}}(H) \delta(y)-V_{\mathrm{IR}}(H)[\delta(y+L)+\delta(y-L)]\right\}+S_{G H},
\end{aligned}
$$

where $R$ is the $5 \mathrm{D}$ Ricci scalar and $H$ is the $\mathrm{SU}(2)$ Higgs doublet. Whereas, $V_{B}(H)$ and $V_{\mathrm{UV}(\mathrm{IR})}(H)$ are the bulk and UV(IR) brane potentials, respectively. Above, the $S_{G H}$ is the Gibbons-Hawking boundary action defined in eq. (2.2).

We can write the $y$-dependent vacuum expectation value (vev) of the SU(2) Higgs doublet as:

$$
\langle H\rangle=\frac{1}{\sqrt{2}}\left(\begin{array}{c}
0 \\
\phi(y)
\end{array}\right) .
$$

In order to find the background solutions we need to solve the coupled scalar-gravity Einstein equations. The equations for the background fields $A(y)$ and $\phi(y)$ following from the above action (2.10) and the metric ansatz (2.9) can be written as,

$$
\begin{aligned}
6 A^{\prime 2} & =\frac{1}{2} \phi^{2}-V_{B}(\phi), \\
3 A^{\prime \prime}+6 A^{\prime 2} & =-\frac{1}{2} \phi^{\prime 2}-V_{B}(\phi)-V_{\mathrm{UV}}(\phi) \delta(y)-V_{\mathrm{IR}}(\phi)[\delta(y+L)+\delta(y-L)], \\
\phi^{\prime \prime}+4 A^{\prime} \phi^{\prime} & =\frac{\partial V_{B}(\phi)}{\partial \phi}+\frac{\partial V_{\mathrm{UV}}(\phi)}{\partial \phi} \delta(y)+\frac{\partial V_{\mathrm{IR}}(\phi)}{\partial \phi}[\delta(y+L)+\delta(y-L)] .
\end{aligned}
$$


Superpotential method. In the following we will layout the so-called superpotential method for solving the above set of coupled scalar-gravity equations [4]. Although the use of this method is motivated by supersymmetry, no supersymmetry is involved in our setup. The method is elegant and very efficient, in particular it applies to the system of second order differential equations (2.12)-(2.14) and reduces them to a set of first order ordinary differential equations which are much easier to deal with. It is assumed that the scalar potential $V_{B}(\phi)$ could be expressed in terms of the superpotential $W(\phi)$ as $[4,46]$,

$$
V_{B}(\phi)=\frac{1}{8}\left(\frac{\partial W(\phi)}{\partial \phi}\right)^{2}-\frac{1}{6} W(\phi)^{2}
$$

where the superpotential $W(\phi)$ satisfies,

$$
\phi^{\prime}=\frac{1}{2} \frac{\partial W(\phi)}{\partial \phi}, \quad A^{\prime}=-\frac{1}{6} W(\phi),
$$

along with the following jump at $y=0$ and boundary conditions $y= \pm L$ :

$$
\begin{aligned}
\frac{1}{2}[W(\phi)]_{0} & =\left.V_{\mathrm{UV}}(\phi)\right|_{\phi=\phi(0)}, & \frac{1}{2}\left[\frac{\partial W(\phi)}{\partial \phi}\right]_{0} & =\left.\frac{\partial V_{\mathrm{UV}}(\phi)}{\partial \phi}\right|_{\phi=\phi(0)} . \\
\left.W(\phi)\right|_{ \pm L} & =-\left.V_{\mathrm{IR}}(\phi)\right|_{\phi=\phi( \pm L)}, & \left.\frac{\partial W(\phi)}{\partial \phi}\right|_{ \pm L} & =-\left.\frac{\partial V_{\mathrm{IR}}(\phi)}{\partial \phi}\right|_{\phi=\phi( \pm L)} .
\end{aligned}
$$

Above, the jump across the UV-brane of $j(y)=W(\phi)$ and $\partial W(\phi) / \partial \phi$, is defined as follows:

$$
[j(y)]_{0} \equiv \lim _{\epsilon \rightarrow 0}\{j(0+\epsilon)-j(0-\epsilon)\} .
$$

Let us consider the following form of the superpotential $W(\phi)$

$$
W(\phi)=\left\{\begin{array}{rlr}
6 k+(2+\beta) k \phi^{2} & \text { for } & 0<y<L \\
-6 k-(2+\beta) k \phi^{2} & \text { for } & -L<y<0
\end{array},\right.
$$

where $\beta \equiv \sqrt{4+\mu_{B}^{2} / k^{2}}$ parameterises the bulk mass $\mu_{B}$ of the Higgs field and $k$ is a constant of order $M_{*}$. The above form of $W(\phi)$ is $\mathbb{Z}_{2}$ odd under $y \rightarrow-y$, which ensures, by the virtue of eq. (2.16), that $A(y)$ and $\phi(y)$ are $\mathbb{Z}_{2}$ even. We get the scalar potential $V_{B}(\phi)$ from eq. $(2.15)$ as

$$
V_{B}(\phi)=-6 k^{2}+\frac{1}{2} \mu_{B}^{2} \phi^{2}-\frac{k^{2}}{2}(2+\beta)^{2} \phi^{4} .
$$

We employ the following forms of the brane-localized potentials,

$$
\begin{gathered}
V_{\mathrm{UV}}(\phi)=W(\phi)+\frac{\lambda_{\mathrm{UV}}}{4 k^{2}}\left(\phi^{2}-\phi_{\mathrm{UV}}^{2}\right)^{2}, \\
V_{\mathrm{IR}}(\phi)=-W(\phi)+\frac{\lambda_{\mathrm{IR}}}{4 k^{2}}\left(\phi^{2}-\phi_{\mathrm{IR}}^{2}\right)^{2},
\end{gathered}
$$

where $\phi_{\mathrm{UV}(\mathrm{IR})}$ is a constant value of the background vev at $y=0( \pm L)$ and $\lambda_{\mathrm{UV}(\mathrm{IR})}$ is the quartic coupling at the UV(IR) brane. The background vev $\phi(y)$ and the warp-function $A(y)$ can be obtained by integrating the first order equations (2.16) as

$$
\phi(y)=\phi_{\mathrm{IR}} e^{(2+\beta) k(|y|-L)},
$$



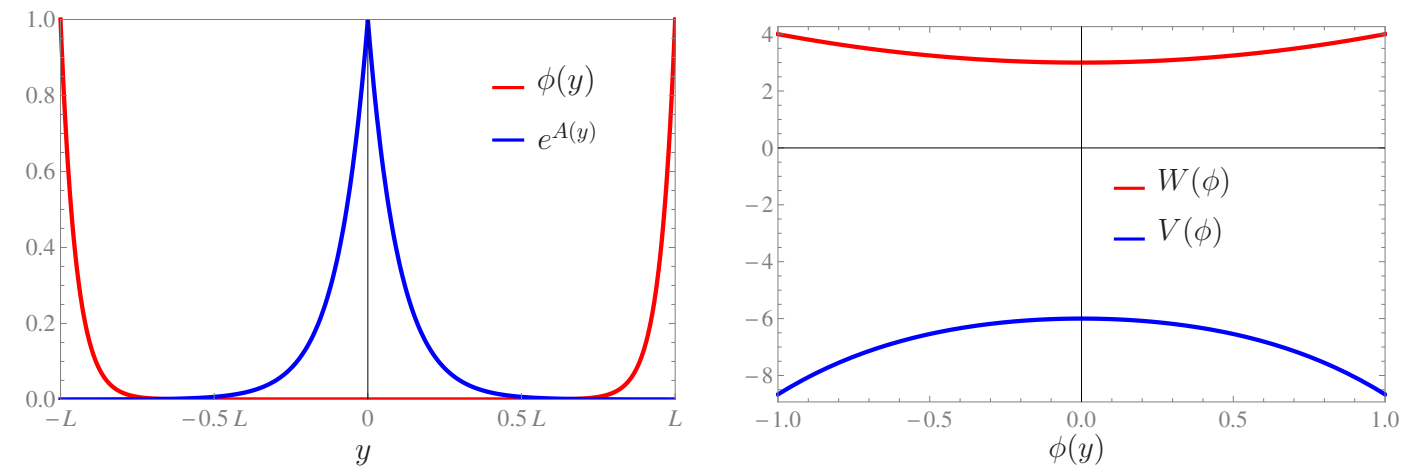

Figure 3. The left graph shows the $y$-dependent background vev $\phi(y)$ and the warp factor $e^{A(y)}$ as function of $y$, while the right graph illustrates the shapes of superpotential $W(\phi)$ and the bulk scalar potential $V_{B}(\phi)$ as a function of background vev $\phi$. The parameter choice adopted for the graphs is: $\beta=0, k=1$ and $\phi_{\mathrm{IR}}=1$.

$$
A(y)=-k|y|-\frac{1}{12} \phi_{\mathrm{IR}}^{2} e^{-2(2+\beta) k L}\left[e^{2(2+\beta) k|y|}-1\right] .
$$

Moreover, we impose the following normalization condition for the background vev:

$$
\int_{-L}^{L} d y e^{2 A(y)} \phi^{2}(y)=v_{S M}^{2}
$$

where $v_{S M}$ is the SM vev. The $\phi_{\mathrm{IR}}$ resulting from the above normalization reads (see also eq. (A.51)):

$$
\phi_{\mathrm{IR}} \simeq v_{S M} \sqrt{k(1+\beta)} e^{k L}
$$

As we will see below in order to solve the gauge hierarchy problem, one needs $k L \simeq 37$ and for $\phi_{\mathrm{IR}} \sim \mathcal{O}\left(M_{\mathrm{Pl}}^{3 / 2}\right), k \approx \mathcal{O}\left(M_{\mathrm{Pl}}\right)$ and $\beta \approx \mathcal{O}(1)$, the above expression implies that then $v_{S M} \sim \mathcal{O}(\mathrm{TeV})$. In figure 3 , we have plotted the $y$-dependent background vev $\phi(y)$ and the warp factor $e^{A(y)}$ as function of $y$ in the left panel, while the right panel shows the superpotential $W(\phi)$ and the bulk scalar potential $V_{B}(\phi)$ as a function of $\phi$.

In order to gain some intuition, it is instructive to count number of parameters and constraints to see how many fine-tunings are necessary to obtain solutions with Minkowski D3-branes. There are three integration constants coming from the first order eqs. (2.15) and (2.16). One of these three, $A(0)$, is trivial additive constant hence we are left with two parameters to be fixed. Let us count the number of constraints: there are two constraints from the jump at $y=0$ from eq. (2.17) and two constraints from the boundaries at $\pm L$ eq. (2.18) (note that the boundary conditions are same at $\pm L$ ). We can integrate eq. (2.15) to fix the integration constant by requiring $W\left(\phi_{\mathrm{UV}}\right)=V_{\mathrm{UV}}\left(\phi_{\mathrm{UV}}\right)$. This requirement together with eq. (2.15) will trivially satisfy the second jump condition of eq. (2.17). We can then integrate the first equation of eq. (2.16) to get $\phi(y)$ and the position of the IRbrane is fixed w.r.t. the UV-brane by requiring $\phi(L)=\phi_{\mathrm{IR}}$. This fixes all the parameters of the theory but we are still left with two constraints eq. (2.18) which are not independent of each other as fixing $W\left(\phi_{\mathrm{IR}}\right)=-V_{\mathrm{IR}}\left(\phi_{\mathrm{IR}}\right)$ will trivially satisfy second equation of eq. (2.18). Hence we are left with precisely one fine-tuning which is required to ensure flat-brane solutions and is a common pathology of RS-like models, see also ref. [4]. 
Let us check that the $4 \mathrm{D}$ cosmological constant $\Lambda_{4}$ is indeed zero after having this fine-tuning is achieved. The $4 \mathrm{D}$ cosmological constant for this scalar-gravity theory (2.10) is given by the following integral calculated for the background solutions

$$
\begin{aligned}
\Lambda_{4}= & -\int_{-L}^{L} d y \sqrt{-g}\left\{\frac{R}{2}-\frac{1}{2} \phi^{\prime 2}-V_{B}(\phi)-V_{\mathrm{UV}}(\phi) \delta(y)-V_{\mathrm{IR}}(\phi)[\delta(y+L)+\delta(y-L)]\right\}+\left.\sqrt{-\hat{g}} \mathcal{K}\right|_{-L} ^{L} \\
=- & \int_{-L}^{L} d y e^{4 A}\left\{6\left(A^{\prime}+\frac{1}{6} W\right)^{2}-\frac{1}{2}\left(\phi^{\prime}-\frac{1}{2} \frac{\partial W}{\partial \phi}\right)^{2}-e^{-4 A} \frac{d}{d y}\left[e^{4 A}\left(4 A^{\prime}+\frac{1}{2} W\right)\right]\right. \\
& \left.+\frac{1}{2} \frac{\partial W}{\partial y}-V_{\mathrm{UV}}(\phi) \delta(y)-V_{\mathrm{IR}}(\phi)[\delta(y+L)+\delta(y-L)]\right\}-\left.4 e^{4 A} A^{\prime}\right|_{-L} ^{L} \\
= & -\int_{-L}^{L} d y e^{4 A}\left\{6\left(A^{\prime}+\frac{1}{6} W\right)^{2}-\frac{1}{2}\left(\phi^{\prime}-\frac{1}{2} \frac{\partial W}{\partial \phi}\right)^{2}\right\} \\
& -\frac{1}{2}[W]_{0}+V_{\mathrm{UV}}[\phi(0)]+\left.\frac{e^{4 A}}{2} W\right|_{-L} ^{L}+\frac{e^{4 A(L)}}{2} V_{\mathrm{IR}}[\phi(L)]+\frac{e^{4 A(L)}}{2} V_{\mathrm{IR}}[\phi(-L)] .
\end{aligned}
$$

Above, in the second line we have only used eq. (2.15) and the value of the intrinsic curvature of the boundary manifolds $\mathcal{K}$. In the last line we have adopted the fact that the superpotential $W(\phi)(2.20)$ is discontinuous at $y=0$, hence the jump at $y=0$ follows from the total derivative term. Now it is straightforward to see in the above equation that the full squares vanish due to eq. (2.16), whereas the last line vanishes due to the jump and boundary conditions (2.17)-(2.18). Hence we conclude that the $4 \mathrm{D}$ cosmological constant on the branes is indeed zero, as anticipated from our metric ansatz (2.9).

The brane separation $L$ can be determined by eq. (2.24) as:

$$
k L=\frac{1}{2+\beta} \ln \left(\frac{\phi_{\mathrm{IR}}}{\phi_{\mathrm{UV}}}\right) .
$$

In order to solve the gauge hierarchy problem, the number of $e$-foldings is required to be as follows

$$
A(0)-A(L) \simeq \ln \left(\frac{M_{\mathrm{Pl}}}{m_{E W}}\right) \approx 37 .
$$

By using the eq. (2.25), we get:

$$
A(0)-A(L)=\frac{1}{2+\beta} \ln \left(\frac{\phi_{\mathrm{IR}}}{\phi_{\mathrm{UV}}}\right)+\frac{1}{12}\left(\phi_{\mathrm{IR}}^{2}-\phi_{\mathrm{UV}}^{2}\right) .
$$

Note that for $\beta \geq 0$, both terms in the above relation can contribute to give the desired number of $e$-foldings. When the first term contributes mostly (weak backreaction), so $\phi_{\mathrm{IR}} \gg$ $\phi_{\mathrm{UV}}$, then $k L \simeq 37$. Whereas, when the major contribution to the number of $e$-foldings comes from the second term (strong backreaction), i.e. $\phi_{\mathrm{UV}} \sim \mathcal{O}(1)$ and $\phi_{\mathrm{IR}} \sim \mathcal{O}(20)$ then $k L \sim \mathcal{O}(1)$. In this paper we consider the weak backreaction scenario, i.e. $k L \simeq 37$, which implies that the second term in the warp-function (2.25) is negligible and hence the form of warp-function is $A(y) \simeq-k|y|$ which is the same as in the previous subsection with no backreaction. Note that the weak backreaction scenario is also required in order to insure the SM vev $v_{S M} \simeq 246 \mathrm{GeV}$ below the KK-scale, see e.g. ref. [21]. The case of strong backreaction is also interesting as the fine-tuning required is much less than that of the weak backreaction scenario but we will not consider it here. 


\subsection{Warped KK-parity}

In this section we employ the background solution for the $\mathbb{Z}_{2}$ symmetric background (IRUV-IR) geometry considered in section 2.1 and show how KK-parity is manifested within this geometric setup. The IR-UV-IR geometry of section 2.1 is $\mathbb{Z}_{2}$-symmetric and we will consider this symmetry to be exact for our $5 \mathrm{D}$ theory. If the $5 \mathrm{D}$ theory has this $\mathbb{Z}_{2}$-parity (symmetry) then the Schrödinger-like potential for all the fields is symmetric, resulting in even (symmetric) and odd (antisymmetric) eigenmodes. Thus, a general field $\Phi(x, y)$ can be KK decomposed as follows

$$
\Phi(x, y)=\sum_{n} \phi_{n}(x) f_{n}(y)
$$

where, due to the $\mathbb{Z}_{2}$ geometry, the wave functions $f_{n}(y)$ are either even or odd, so that

$$
\Phi(x, y) \equiv \Phi^{( \pm)}(x, y)
$$

with

$$
\begin{aligned}
& \Phi^{(+)}(x, y)=\sum_{n} \phi_{n}^{(+)}(x) f_{n}^{(+)}(y) \stackrel{y \rightarrow-y}{\longrightarrow}+\Phi^{(+)}(x, y), \\
& \Phi^{(-)}(x, y)=\sum_{n} \phi_{n}^{(-)}(x) f_{n}^{(-)}(y) \stackrel{y \rightarrow-y}{\longrightarrow}-\Phi^{(-)}(x, y) .
\end{aligned}
$$

Due to the geometric $\mathbb{Z}_{2}$ symmetry, a single odd KK-mode cannot couple to two even KK-modes in the $4 \mathrm{D}$ effective theory, therefore the lowest odd KK-mode will be stable and may serve as a dark matter candidate.

Furthermore, as the geometry is $\mathbb{Z}_{2}$ symmetric in $y \in[-L, L]$, the continuity conditions for odd and even modes at $y=0$ strongly impact the physics scenario. Our choice will be that the odd (even) modes satisfy Dirichlet (Neumann or mixed) boundary (jump) conditions (b.c.) at $y=0$, respectively. As for the odd modes, continuity implies that they must be zero at $y=0$, but we could also have demanded the Neumann conditions that their $y$ derivative be zero at $y=0$. We choose not to impose this additional b.c. in this work. As regards the even modes, one cannot choose Dirichlet b.c. at $y=0$ because of the presence of the UV-brane and associated "jump" conditions following from the equations of motion.

\section{SM EWSB by bulk Higgs doublet - the truncated-inert-doublet model}

In this section we consider all the SM fields in the bulk and study phenomenological implications of our symmetric geometry. Hereafter, we consider only the weak backreaction scenario discussed in section 2.2 and we employ the metric (1.1) as a solution of the IRUV-IR geometric background. Note also that vev of effective 4D Higgs field is of the electroweak scale which is much smaller than the gravity (Planck mass) scale (see eq. (2.27) and discussion below), therefore their back-reaction on the background geometry would be negligible, see sub-section 2.2 and refs. [21, 25]. Moreover, we follow closely the Abelian 
case A.1 for EWSB of the 5D SM gauge group and as shown in appendix A this approach is equivalent, at the zero-mode level, to the canonical approach (appendix A.2), i.e. when the bulk Higgs field is expanded around the background vev.

The $5 \mathrm{D}$ action for the electroweak sector of the SM can be written $\operatorname{as}^{5}$

$$
\begin{aligned}
S=-\int d^{5} x \sqrt{-g}\{ & \frac{1}{4} F_{M N}^{a} F^{a M N}+\frac{1}{4} B_{M N} B^{M N}+\left|D_{M} H\right|^{2}+\mu_{B}^{2}|H|^{2} \\
& \left.+V_{\mathrm{IR}}(H) \delta(y+L)+V_{\mathrm{UV}}(H) \delta(y)+V_{\mathrm{IR}}(H) \delta(y-L)\right\},
\end{aligned}
$$

where $F_{M N}^{a}$ and $B_{M N}$ are the $5 \mathrm{D}$ field strength tensors for $\mathrm{SU}(2)$ and $\mathrm{U}(1)_{Y}$, respectively with $a$ being the number generators of $\mathrm{SU}(2)$. Above, $H$ is the $\mathrm{SU}(2)$ doublet and its brane potentials are

$$
V_{\mathrm{UV}}(H)=\frac{m_{\mathrm{UV}}^{2}}{k}|H|^{2}, \quad V_{\mathrm{IR}}(H)=-\frac{m_{\mathrm{IR}}^{2}}{k}|H|^{2}+\frac{\lambda_{\mathrm{IR}}}{k^{2}}|H|^{4} .
$$

In our approach, we do not put the Higgs quartic terms in the bulk nor on the UV-brane since we want EWSB to take place near the IR-brane. The covariant derivative $D_{M}$ is defined as follows:

$$
D_{M}=\partial_{M}-i \frac{g_{5}}{2} \tau^{a} A_{M}^{a}-i \frac{g_{5}^{\prime}}{2} B_{M},
$$

where $\tau^{a}$ are Pauli matrices and $g_{5}\left(g_{5}^{\prime}\right)$ is the coupling constant for the $A_{M}^{a}\left(B_{M}\right)$ fields.

There is an important comment in order here concerning the above particular forms of bulk and brane-localized potentials. We have dropped quartic terms in the UV and the bulk potentials, even though there is no symmetry that would protect this choice. However there are phenomenological arguments that support such an option. As one can notice the UV Higgs quartic operator, i.e. $V_{\mathrm{UV}}(H) \supset \lambda_{\mathrm{UV}} / k^{2}|H|^{4}$ is highly suppressed as $\lambda_{\mathrm{UV}} / k^{2} \sim$ $\mathcal{O}\left(M_{\mathrm{Pl}}^{-2}\right)$. Whereas for the IR Higgs quartic operator, suppression of $\lambda_{\mathrm{IR}} / k^{2}$ is reduced to $\sim \mathcal{O}\left(m_{K K}^{-2}\right)$ due to the non-trivial warp factor at the IR-brane, see also refs. $[22,26]$. Similarly, the bulk quartic term would also be suppressed by some intermediate scale. For simplicity we ignore those terms. We shell emphasize that the reasoning behind ignoring the bulk and the UV-brane localized quartic terms is purely phenomenological in its nature. It is also worth recalling that in this work we are going to investigate the possibility of providing a dark matter candidate as a lowest mass odd scalar KK mode. It is fair to expect that those results will not receive any substantial corrections in the presence of quartic UV and brane terms. Besides that, this kind of phenomenological approximation of ignoring the bulk and UV-brane quartic terms is widely used in the literature on bulk Higgs scenarios in RS1-like models, see for example [15-26, 28].

Note also that due to the $\mathbb{Z}_{2}$ geometric symmetry, physics of the full IR-UV-IR setup can be described completely by a single copy of RS1, i.e. UV-IR setup, however in that

\footnotetext{
${ }^{5}$ Note that the Higgs part of the Lagrangian is not exactly same as considered in section 2.2 for the analysis of the backreaction, here we neglect the quartic terms in the bulk and UV-brane potentials solely for phenomenological convenience since these terms are suppressed, see the main text below. Moreover, we introduce some convenient and familiar notations for brane potential parameters.
} 
scenario each bulk field would be a subject of Neumann (or mixed) and Dirichlet boundary conditions at $y=0$ for even and odd fields, respectively.

It is instructive to make the usual redefinition of the gauge fields,

$$
\begin{aligned}
W_{M}^{ \pm}(x, y) & \equiv \frac{1}{\sqrt{2}}\left(A_{M}^{1} \mp i A_{M}^{2}\right), \\
Z_{M}(x, y) & \equiv \frac{1}{\sqrt{g_{5}^{2}+g_{5}^{\prime 2}}}\left(g_{5} A_{M}^{3}-g_{5}^{\prime} B_{M}\right), \\
A_{M}(x, y) & \equiv \frac{1}{\sqrt{g_{5}^{2}+g_{5}^{\prime 2}}}\left(g_{5}^{\prime} A_{M}^{3}+g_{5} B_{M}\right) .
\end{aligned}
$$

Analogous to the $4 \mathrm{D}$ procedure, we define the 5D Weinberg angle $\theta$ as follows:

$$
\cos \theta \equiv \frac{g_{5}}{\sqrt{g_{5}^{2}+g_{5}^{\prime 2}}}, \quad \sin \theta \equiv \frac{g_{5}^{\prime}}{\sqrt{g_{5}^{2}+g_{5}^{\prime 2}}}
$$

The 5 D gauge fields corresponding to the gauge group $\mathrm{SU}(2) \times \mathrm{U}(1)_{Y}$ are then

$$
\mathbb{A}_{M}(x, y) \equiv\left(\begin{array}{cc}
\sin \theta A_{M}+\frac{\cos ^{2} \theta-\sin ^{2} \theta}{2 \cos \theta} Z_{M} & \frac{1}{\sqrt{2}} W_{M}^{+} \\
\frac{1}{\sqrt{2}} W_{M}^{-} & -\frac{1}{2 \cos \theta} Z_{M}
\end{array}\right) .
$$

The gauge transformations for the Higgs doublet $H(x, y)$ and gauge matrix $\mathbb{A}_{M}$ under the gauge group $\mathrm{SU}(2) \times \mathrm{U}(1)_{Y}$ can be written as

$$
\begin{aligned}
H(x, y) \rightarrow H^{\prime}(x, y) & =\mathrm{U}(x, y) H(x, y), \\
\mathbb{A}_{M}(x, y) \rightarrow \mathbb{A}_{M}^{\prime}(x, y) & =\mathrm{U}(x, y) \mathbb{A}_{M}(x, y) U^{-1}(x, y)-\frac{i}{g_{5}}\left(\partial_{M} \mathrm{U}(x, y)\right) U^{-1}(x, y),
\end{aligned}
$$

where $\mathrm{U}(x, y)$ is the unitary matrix corresponding to the fundamental representation of $\mathrm{SU}(2) \times \mathrm{U}(1)_{Y}$ gauge transformations.

We will choose the 5D axial gauge analogous to the Abelian case A.1 by taking $\mathbb{A}_{5}(x, y)=0$. Note that we can always find $\mathrm{U}(x, y)$ such that the axial gauge is manifest, i.e. $\mathbb{A}_{5}(x, y)=0$. We employ an axial gauge choice for the non-Abelian case of the form

$$
\mathrm{U}(x, y)=\widehat{\mathrm{U}}(x) \mathcal{P} e^{-i g_{5} \int_{0}^{y} d y^{\prime} \mathbb{A}_{5}\left(x, y^{\prime}\right)},
$$

where $\widehat{\mathrm{U}}(x)$ is the residual $4 \mathrm{D}$ gauge transformation and $\mathcal{P}$ denotes path-ordering of the exponential. Another key point for the later discussion is that this $4 \mathrm{D}$ residual gauge transformation $\widehat{\mathrm{U}}(x)$ is independent of $y$ and thus even under the geometric parity.

As we have demonstrated in appendix A, due to the symmetric geometry the background fields in the IR-UV-IR setup separate into even and odd bulk wave functions. Hence, it is straightforward to generalize the results obtained in appendix A.1 for the Abelian model to the electroweak sector of the SM. Let us start by decomposing the Higgs doublet and gauge fields into components of definite parity as follows:

$$
H(x, y)=H^{(+)}(x, y)+H^{(-)}(x, y), \quad V_{M}(x, y)=V_{M}^{(+)}(x, y)+V_{M}^{(-)}(x, y),
$$


where $V_{M} \equiv\left(A_{M}, W_{M}^{ \pm}, Z_{M}\right)$. We can write the action (3.1) up to quadratic level in the $\mathbb{A}_{5}(x, y)=0$ gauge as

$$
\begin{aligned}
S^{(2)}= & -\int d^{5} x \sqrt{-g}\left\{\frac{1}{2} \mathcal{W}_{(+) \mu \nu}^{+} \mathcal{W}_{(+)}^{-\mu \nu}+\partial_{5} W_{(+) \mu}^{+} \partial^{5} W_{(+)}^{-\mu}+\frac{1}{4} \mathcal{Z}_{\mu \nu}^{(+)} \mathcal{Z}_{(+)}^{\mu \nu}+\frac{1}{2} \partial_{5} Z_{\mu}^{(+)} \partial^{5} Z_{(+)}^{\mu}\right. \\
& +\frac{1}{2} \mathcal{W}_{(-) \mu \nu}^{+} \mathcal{W}_{(-)}^{-\mu \nu}+\partial_{5} W_{(-) \mu}^{+} \partial^{5} W_{(-)}^{-\mu}+\frac{1}{4} \mathcal{Z}_{\mu \nu}^{(-)} \mathcal{Z}_{(-)}^{\mu \nu}+\frac{1}{2} \partial_{5} Z_{\mu}^{(-)} \partial^{5} Z_{(-)}^{\mu} \\
& +\frac{1}{4} \mathcal{F}_{\mu \nu}^{(+)} \mathcal{F}_{(+)}^{\mu \nu}+\frac{1}{2} \partial_{5} A_{\mu}^{(+)} \partial_{5} A_{(+)}^{\mu}+\frac{1}{4} \mathcal{F}_{\mu \nu}^{(-)} \mathcal{F}_{(-)}^{\mu \nu}+\frac{1}{2} \partial_{5} A_{\mu}^{(-)} \partial^{5} A_{(-)}^{\mu} \\
& +\mathbb{D}_{M} H^{(+) \dagger} \mathbb{D}^{M} H^{(+)}+\mu_{B}^{2}\left|H^{(+)}\right|^{2}+\mathbb{D}_{M} H^{(-) \dagger} \mathbb{D}^{M} H^{(-)}+\mu_{B}^{2}\left|H^{(-)}\right|^{2} \\
& \left.+\frac{m_{\mathrm{UV}}^{2}}{k}\left|H^{(+)}\right|^{2} \delta(y)-\frac{m_{\mathrm{IR}}^{2}}{k}\left(\left|H^{(+)}\right|^{2}+\left|H^{(-)}\right|^{2}\right)[\delta(y+L)+\delta(y-L)]\right\}
\end{aligned}
$$

where we have adopted the following definitions:

$$
\begin{aligned}
& \tilde{\mathcal{V}}_{\mu \nu}^{( \pm)} \equiv \partial_{\mu} \tilde{V}_{\nu}^{( \pm)}-\partial_{\nu} \tilde{V}_{\mu}^{( \pm)}, \quad \mathcal{F}_{\mu \nu}^{( \pm)} \equiv \partial_{\mu} A_{\nu}^{( \pm)}-\partial_{\nu} A_{\mu}^{( \pm)}, \\
& \mathbb{D}_{\mu}\left(\begin{array}{l}
H^{(+)} \\
H^{(-)}
\end{array}\right) \equiv\left[\partial_{\mu}-i g_{5}\left(\begin{array}{cc}
\mathbb{A}_{\mu}^{(+)} & \mathbb{A}_{\mu}^{(-)} \\
\mathbb{A}_{\mu}^{(-)} & \mathbb{A}_{\mu}^{(+)}
\end{array}\right)\right]\left(\begin{array}{l}
H^{(+)} \\
H^{(-)}
\end{array}\right), \\
& \mathbb{D}_{5}\left(\begin{array}{l}
H^{(+)} \\
H^{(-)}
\end{array}\right) \equiv\left[\partial_{5}-i g_{5}\left(\begin{array}{ll}
\mathbb{A}_{5}^{(-)} & \mathbb{A}_{5}^{(+)} \\
\mathbb{A}_{5}^{(+)} & \mathbb{A}_{5}^{(-)}
\end{array}\right)\right]\left(\begin{array}{l}
H^{(+)} \\
H^{(-)}
\end{array}\right),
\end{aligned}
$$

where $\tilde{V}_{\mu} \equiv\left(W_{\mu}^{ \pm}, Z_{\mu}\right)$ and $\mathbb{A}_{M}^{( \pm)}$was defined in (3.7).

It is convenient to write the Higgs doublets in the following form:

$$
\left(\begin{array}{l}
H^{(+)} \\
H^{(-)}
\end{array}\right)=e^{i g_{5}\left(\Pi^{(+)} \mathbb{1}+\Pi^{(-)} \tau_{1}\right)}\left(\begin{array}{l}
\mathcal{H}^{(+)} \\
\mathcal{H}^{(-)}
\end{array}\right),
$$

where $\mathcal{H}$ and $\Pi$ are defined as (the parity indices are suppressed)

$$
\begin{aligned}
\mathcal{H}(x, y) & \equiv \frac{1}{\sqrt{2}}\left(\begin{array}{c}
0 \\
h(x, y)
\end{array}\right), \\
\Pi(x, y) & \equiv\left(\begin{array}{cc}
\frac{\cos ^{2} \theta-\sin ^{2} \theta}{2 \cos \theta} \pi_{Z} & \frac{1}{\sqrt{2}} \pi_{W}^{+} \\
\frac{1}{\sqrt{2}} \pi_{W}^{-} & -\frac{1}{2 \cos \theta} \pi_{Z}
\end{array}\right) .
\end{aligned}
$$

We KK-decompose the Higgs doublets $H^{( \pm)}(x, y)$ and the gauge fields $V_{\mu}^{( \pm)}(x, y)$ as

$$
\begin{aligned}
\mathcal{H}^{( \pm)}(x, y) & =\sum_{n} \mathcal{H}_{n}^{( \pm)}(x) f_{n}^{( \pm)}(y) \\
\pi_{\tilde{V}}^{( \pm)}(x, y) & =\sum_{n} \pi_{\tilde{V} n}^{( \pm)}(x) a_{\tilde{V} n}^{( \pm)}(y) \\
V_{\mu}^{( \pm)}(x, y) & =\sum_{n} V_{\mu n}^{( \pm)}(x) a_{V_{n}}^{( \pm)}(y)
\end{aligned}
$$


where the wave-functions $f_{n}^{( \pm)}(y)$ and $a_{V_{n}}^{( \pm)}(y)$ satisfy

$$
\begin{aligned}
-\partial_{5}\left(e^{4 A(y)} \partial_{5} f_{n}^{( \pm)}(y)\right)+\mu_{B}^{2} e^{4 A(y)} f_{n}^{( \pm)}(y) & =m_{n}^{2( \pm)} e^{2 A(y)} f_{n}^{( \pm)}(y), \\
-\partial_{5}\left(e^{2 A(y)} \partial_{5} a_{V_{n}}^{( \pm)}(y)\right) & =m_{V_{n}^{( \pm)}}^{2} a_{V_{n}}^{( \pm)}(y),
\end{aligned}
$$

and, with our background geometry $A(y)=-k|y|$. The $y$-dependent wave functions $f_{n}^{( \pm)}(y)$ and $a_{V_{n}}^{( \pm)}(y)$ satisfy the following orthonormality conditions:

$$
\int_{-L}^{+L} d y e^{2 A(y)} f_{m}^{( \pm)}(y) f_{n}^{( \pm)}(y)=\delta_{m n}, \quad \int_{-L}^{+L} d y a_{V_{m}}^{( \pm)}(y) a_{V_{n}}^{( \pm)}(y)=\delta_{m n}
$$

The even modes are subject to jump conditions at $y=0$ while the odd modes are constrained by continuity at $y=0$, resulting in the following boundary conditions:

$$
\begin{aligned}
\left.\left(\partial_{5}-\frac{m_{\mathrm{UV}}^{2}}{k}\right) f_{n}^{(+)}(y)\right|_{0}=0, & \left.f_{n}^{(-)}(y)\right|_{0}=0, \\
\left.\partial_{5} a_{V_{n}}^{(+)}(y)\right|_{0}=0, & \left.a_{V_{n}}^{(-)}(y)\right|_{0}=0 .
\end{aligned}
$$

The b.c. at $y= \pm L$ are:

$$
\left.\left( \pm \partial_{5}-\frac{m_{\mathrm{IR}}^{2}}{k}\right) f_{n}^{( \pm)}(y)\right|_{ \pm L}=0,\left.\quad \partial_{5} a_{V_{n}}^{( \pm)}(y)\right|_{ \pm L}=0
$$

As pointed out in the Abelian case A.1, the choices of b.c. for $a_{n}^{(+)}(y)$ at $y=0, \pm L$ are motivated by the requirement that the even zero-mode profiles for gauge bosons be non-zero.

It is worth mentioning here that the choice of writing the Higgs doublets $H^{( \pm)}$in the form of eq. (3.16) and using the KK decomposition for the pseudoscalars $\pi_{\tilde{V}}^{( \pm)}$as given in eq. (3.20) are both motivated by model-building considerations discussed below. The other possibility is to choose different KK bases and b.c. for the pseudoscalars $\pi_{\tilde{V}}^{( \pm)}$such that after SSB these pseudoscalars become Nambu-Goldstone bosons (NGB). The even zero-mode gauge bosons would then acquire masses by eating up the even-parity NGB, whereas the odd-parity NGB would remain in the spectrum (the odd zero-mode gauge boson fields being zero, see below). An effective potential for the odd-parity NGB would be generated through their interactions with gauge bosons, hence making them pseudoNGB. We don't follow this approach here but it is an interesting possibility in which the neutral odd pseudo-NGB would be a composite dark Higgs in the dual CFT description. ${ }^{6}$

We assume that the KK-scale is high enough, i.e. $m_{K K} \sim \mathcal{O}$ (few) $\mathrm{TeV}$, that we can consider an effective theory where only the lowest modes (zero-modes with masses much below $\left.m_{K K}\right)$ are kept. It is important to note that the odd zero-mode wave functions obey $a_{V_{0}}^{(-)}(y)=0$, as can be easily seen from eq. (3.23) along with the b.c. (3.26) and (3.27). As a consequence of $a_{V_{0}}^{(-)}(y)=0$, the odd zero-mode gauge fields $V_{0 \mu}^{(-)}(x)$ and the odd Goldstone

\footnotetext{
${ }^{6}$ At the final stages of the present work, ref. [47] appeared where the authors considered composite dark sectors. A similar construction can be naturally realized as a CFT dual to the model considered here.
} 
modes $\pi_{\tilde{V}_{0}}^{(-)}(x)$ will not be present in the effective $4 \mathrm{D}$ theory. Moreover, the even zero-mode gauge profile is constant, i.e. $a_{V_{0}}^{(+)}(y)=1 / \sqrt{2 L}$. Using the results from appendix A, we can determine the values of the couplings and mass parameters in the effective $4 \mathrm{D}$ theory in terms of the parameters of the fundamental $5 \mathrm{D}$ theory. The result is that we can write down the effective $4 \mathrm{D}$ action for the zero-modes as

$$
\begin{aligned}
S_{\text {eff }}^{(2)}= & -\int d^{4} x\left\{\frac{1}{4} \mathcal{F}_{\mu \nu}^{0(+)} \mathcal{F}_{0(+)}^{\mu \nu}+\frac{1}{4} \mathcal{Z}_{\mu \nu}^{0(+)} \mathcal{Z}_{0(+)}^{\mu \nu}+\frac{1}{2} \mathcal{W}_{\mu \nu}^{+0(+)} \mathcal{W}_{0(+)}^{-\mu \nu}+\partial_{\mu} \mathcal{H}_{0}^{(+) \dagger} \partial^{\mu} \mathcal{H}_{0}^{(+)}\right. \\
& +\partial_{\mu} \mathcal{H}_{0}^{(-) \dagger} \partial^{\mu} \mathcal{H}_{0}^{(-)}+m_{0}^{2(+)}\left|\mathcal{H}_{0}^{(+)}\right|^{2}+m_{0}^{2(-)}\left|\mathcal{H}_{0}^{(-)}\right|^{2}-i g_{4} \partial_{\mu} \mathcal{H}_{0}^{(+) \dagger} \mathbb{M}^{\mu} \mathcal{H}_{0}^{(+)} \\
& \left.+i g_{4} \mathcal{H}_{0}^{(+) \dagger} \mathbb{M}_{\mu}^{\dagger} \partial^{\mu} \mathcal{H}_{0}^{(+)}+g_{4}^{2} \mathcal{H}_{0}^{(+) \dagger} \mathbb{M}_{\mu}^{\dagger} \mathbb{M}^{\mu} \mathcal{H}_{0}^{(+)}+g_{4}^{2} \mathcal{H}_{0}^{(-) \dagger} \mathbb{M}_{\mu}^{\dagger} \mathbb{M}^{\mu} \mathcal{H}_{0}^{(-)}\right\}
\end{aligned}
$$

where $\mathbb{M}_{\mu}$ is defined as

$$
\mathbb{M}_{\mu} \equiv \mathbb{U}^{\dagger} \hat{\mathbb{A}}_{0 \mu}^{(+)} \mathbb{U}+\frac{i}{g_{4}} \mathbb{U}^{\dagger} \partial_{\mu} \mathbb{U},
$$

with $\mathbb{U} \equiv e^{i g_{4} \widehat{\Pi}_{0}^{(+)}}$and $g_{4} \equiv g_{5} / \sqrt{2 L}$. In the above action $\mathcal{H}_{0}^{( \pm)}$are real doublets defined in eq. (3.17), implying that $\mathcal{H}_{0}^{( \pm) \dagger}=\mathcal{H}_{0}^{( \pm) \top}$, whereas $\hat{\mathbb{A}}_{0 \mu}^{(+)}$and $\widehat{\Pi}_{0}^{(+)}$are defined as (below we suppress the parity indices and zero-mode index):

$$
\begin{aligned}
& \widehat{\mathbb{A}}_{\mu}(x) \equiv\left(\begin{array}{cc}
\sin \theta A_{\mu}+\frac{\cos ^{2} \theta-\sin ^{2} \theta}{2 \cos \theta} Z_{\mu} & \frac{1}{\sqrt{2}} W_{\mu}^{+} \\
\frac{1}{\sqrt{2}} W_{\mu}^{-} & -\frac{1}{2 \cos \theta} Z_{\mu}
\end{array}\right), \\
& \widehat{\Pi}(x) \equiv\left(\begin{array}{cc}
\frac{\cos ^{2} \theta-\sin ^{2} \theta}{2 \cos \theta} \pi_{Z} & \frac{1}{\sqrt{2}} \pi_{W}^{+} \\
\frac{1}{\sqrt{2}} \pi_{W}^{-} & -\frac{1}{2 \cos \theta} \pi_{Z}
\end{array}\right) .
\end{aligned}
$$

It is important to comment here that the above action is manifestly gauge invariant under the following $\mathrm{SU}(2) \times \mathrm{U}(1)_{Y}$ gauge transformation,

$$
\hat{\mathbb{A}}_{\mu}^{(+)} \rightarrow \widehat{U}_{\hat{\mathbb{A}}_{\mu}^{(+)}} \widehat{U}^{\dagger}-\frac{i}{g_{4}}\left(\partial_{\mu} \widehat{U}\right) \widehat{U}^{\dagger}, \quad \mathbb{U} \rightarrow \widehat{U} e^{i g_{4} \widehat{\Pi}^{(+)}}
$$

whereas the $\mathcal{H}_{0}^{( \pm)}$are gauge invariant under the $4 \mathrm{D}$ residual gauge transformation $\widehat{U}$. Equation (3.28) is a non-Abelian analog of the Abelian zero-mode action given by (A.25).

We introduce a convenient notion for our effective theory by redefining $V_{0 \mu}^{(+)}(x) \equiv$ $V_{\mu}(x), \pi_{\tilde{V} 0}^{(+)}(x) \equiv \pi_{\tilde{V}}(x), \widehat{\Pi}_{0}^{(+)}(x) \equiv \widehat{\Pi}(x)$ and

$$
H_{1}(x) \equiv e^{i g_{4} \widehat{\Pi}(x)} \mathcal{H}_{0}^{(+)}(x), \quad H_{2}(x) \equiv e^{i g_{4} \widehat{\Pi}(x)} \mathcal{H}_{0}^{(-)}(x) .
$$

Now the above effective action (3.28), including the scalar interaction terms, can be written in a nice gauge covariant form as ${ }^{7}$

$$
\begin{aligned}
S_{\text {eff }}=-\int d^{4} x & \left\{\frac{1}{4} \mathcal{F}_{\mu \nu} \mathcal{F}^{\mu \nu}+\frac{1}{4} \mathcal{Z}_{\mu \nu} \mathcal{Z}^{\mu \nu}+\frac{1}{2} \mathcal{W}_{\mu \nu}^{+} \mathcal{W}^{-\mu \nu}\right. \\
& \left.+\left(\mathcal{D}_{\mu} H_{1}\right)^{\dagger} \mathcal{D}^{\mu} H_{1}+\left(\mathcal{D}_{\mu} H_{2}\right)^{\dagger} \mathcal{D}^{\mu} H_{2}+V\left(H_{1}, H_{2}\right)\right\},
\end{aligned}
$$

\footnotetext{
${ }^{7}$ Note that the action of eq. (3.34) is a non-Abelian version of the Abelian zero-mode action (A.29).
} 
where the scalar potential can be written as

$$
V\left(H_{1}, H_{2}\right)=-\mu^{2}\left|H_{1}\right|^{2}-\mu^{2}\left|H_{2}\right|^{2}+\lambda\left|H_{1}\right|^{4}+\lambda\left|H_{2}\right|^{4}+6 \lambda\left|H_{1}\right|^{2}\left|H_{2}\right|^{2} .
$$

The covariant derivative $\mathcal{D}_{\mu}$ is defined as

$$
\mathcal{D}_{\mu}=\partial_{\mu}-i g_{4} \hat{\mathbb{A}}_{\mu}
$$

where $\hat{\mathbb{A}}_{\mu}$ is defined in eq. (3.30). In the above scalar potential the mass parameter $\mu$ and quartic coupling $\lambda$ are defined as (see appendix A),

$$
\mu^{2} \equiv-m_{0}^{2( \pm)}=(1+\beta) m_{K K}^{2} \delta_{\mathrm{IR}}, \quad \lambda \equiv \lambda_{\mathrm{IR}}(1+\beta)^{2},
$$

where $\delta_{\mathrm{IR}}, m_{K K}$ and $\beta$ are defined in eq. (A.26).

Concerning the symmetries of the above potential, one can notice that $V\left(H_{1}, H_{2}\right)$ is invariant under $\left[\mathrm{SU}(2) \times \mathrm{U}(1)_{Y}\right]^{\prime} \times\left[\mathrm{SU}(2) \times \mathrm{U}(1)_{Y}\right]$, where one of the blocks has been gauged while the other one survived as a global symmetry. The zero-modes of the four odd vector bosons $\left(W_{0 \mu}^{(-) \pm}, Z_{0 \mu}^{(-)}\right.$and $\left.A_{0 \mu}^{(-)}\right)$and the three would-be-Goldstone bosons $\Pi_{0}^{(-)}$ have been removed by appropriate b.c., implying that the corresponding gauge symmetry has been broken explicitly. What remains is the truncated inert doublet model, that contains $H_{1,2}$, and the corresponding residual $\mathrm{SU}(2) \times \mathrm{U}(1)_{Y}$ global symmetry of the action. Symmetry under the above mentioned $\mathrm{U}(1)^{\prime} \times \mathrm{U}(1)$ implies in particular that $V\left(H_{1}, H_{2}\right)$ is also invariant under various $\mathbb{Z}_{2}$ 's, for example $H_{1} \rightarrow-H_{1}, H_{2} \rightarrow-H_{2}$ and $H_{1} \rightarrow \pm H_{2}$.

As explained in the Abelian case A.1, we choose the vacuum such that the even parity Higgs field $H_{1}$ acquires a vev, whereas the odd parity Higgs field $H_{2}$ does not, i.e.

$$
v_{1}^{2} \equiv v^{2}=\frac{\mu^{2}}{\lambda}, \quad v_{2}=0 .
$$

Let us now consider fluctuations around the vacuum of our choice

$$
H_{1}(x)=\frac{1}{\sqrt{2}} e^{i g_{4} \widehat{\Pi}}\left(\begin{array}{c}
0 \\
v+h
\end{array}\right), \quad H_{2}(x)=\frac{1}{\sqrt{2}} e^{i g_{4} \widehat{\Pi}}\left(\begin{array}{l}
0 \\
\chi
\end{array}\right),
$$

where $\widehat{\Pi}$ (defined in eq. (3.31)) contains the pseudoscalar Goldstone bosons $\pi_{W^{ \pm}, Z}$. We choose the unitary gauge in which $\pi_{W^{ \pm}, Z}$ are gauged away, that is they are eaten up by the massive gauge bosons $W_{\mu}^{ \pm}$and $Z_{\mu}$. Hence in the unitary gauge our effective action up to the quadratic order in fluctuations is

$$
\begin{aligned}
S_{\text {eff }}^{(2)}=-\int d^{4} x & \left\{\frac{1}{2} \mathcal{W}_{\mu \nu}^{+} \mathcal{W}^{-\mu \nu}+\frac{1}{4} \mathcal{Z}_{\mu \nu} \mathcal{Z}^{\mu \nu}+\frac{1}{4} \mathcal{F}_{\mu \nu} \mathcal{F}^{\mu \nu}+m_{W}^{2} W_{\mu}^{+} W^{-\mu}+\frac{1}{2} m_{Z}^{2} Z_{\mu} Z^{\mu}\right. \\
& \left.+\frac{1}{2} \partial_{\mu} h \partial^{\mu} h+\frac{1}{2} m_{h}^{2} h^{2}+\frac{1}{2} \partial_{\mu} \chi \partial^{\mu} \chi+\frac{1}{2} m_{\chi}^{2} \chi^{2}\right\}
\end{aligned}
$$

where the masses are,

$$
m_{h}^{2}=m_{\chi}^{2}=2 \mu^{2}, \quad m_{W}^{2}=\frac{1}{4} g_{4}^{2} \frac{\mu^{2}}{\lambda}, \quad m_{Z}^{2}=\frac{1}{4}\left(g_{4}^{2}+g_{4}^{\prime 2}\right) \frac{\mu^{2}}{\lambda}=\frac{m_{W}^{2}}{\cos ^{2} \theta_{W}} .
$$


It is worth noticing here that the Higgs mass $m_{h}$ and the dark scalar mass $m_{\chi}$ are degenerate at the tree level. However, as we demonstrate below, this degeneracy is lifted by the quantum corrections predicted by the effective theory below the KK-mass scale. The interaction terms for effective theory can be written as

$$
\begin{aligned}
S_{\mathrm{int}}= & -\int d^{4} x\left\{\lambda v h^{3}+\frac{\lambda}{4} h^{4}+\frac{\lambda}{4} \chi^{4}+3 \lambda v h \chi^{2}+\frac{3}{2} \lambda h^{2} \chi^{2}+\frac{g_{4}^{2}}{2} v W_{\mu}^{+} W^{-\mu} h\right. \\
& \left.+\frac{g_{4}^{2}}{4} W_{\mu}^{+} W^{-\mu}\left(h^{2}+\chi^{2}\right)+\frac{1}{4}\left(g_{4}^{2}+g_{4}^{\prime 2}\right) v h Z_{\mu} Z^{\mu}+\frac{1}{8}\left(g_{4}^{2}+g_{4}^{\prime 2}\right) Z_{\mu} Z^{\mu}\left(h^{2}+\chi^{2}\right)\right\},
\end{aligned}
$$

where we have omitted terms involving gauge interactions alone as they are irrelevant to our discussion below.

\subsection{Quantum corrections to scalar masses}

In this subsection we will study the quantum corrections to the tree-level scalar masses of the Higgs boson $h$ and the dark matter candidate $\chi$.

Before proceeding further, we want to point out here that in this work we have not studied fermions in our geometric setup since our focus is on the bosonic sector of the SM and EWSB. For the sake of self-consistency, we mention here three possibilities for fermion localization and their implications in our geometric setup:

1. In this first scenario, one takes the heavy (top) quarks to be localized towards the IR-brane, while the light quarks and leptons are localized towards the UV-brane. Through this geometric localization one can address the fermion mass hierarchy problem. In this scenario the even and odd zero-modes corresponding to the heavy quarks will be almost degenerate in our symmetric geometry, whereas the odd zero-modes corresponding to the light quarks could be much heavier than their corresponding even zero-modes $[42,43]$.

2. In the second scenario, all the fermions have flat zero-mode profiles. This can be achieved by the choice of appropriate bulk mass parameters for the fermions. As a consequence of flat profiles the odd fermion zero-modes have to disappear and the even zero-modes will correspond to the SM fermions (in this case the fermion mass hierarchy problem is reintroduced).

3. In the third scenario all the fermions are localized towards UV-brane. In this case the masses of all odd zero-modes of the fermions could be heavier than their corresponding even zero-modes.

In this study we implicitly limit ourselves to the last two cases in order that the dark Higgs be the lightest odd particle and all the other odd zero-modes are either not present in our low-energy effective theory or they are much heavier than the dark Higgs, which will therefore be the only relevant dark matter candidate. For either of the choices 2 . or 3 . above, the top Yukawa coupling $y_{t}$ in the low-energy effective theory will be the same as in the SM and the top-quark loop correction to the SM Higgs boson mass will be exactly as in the SM up to the KK cutoff. In case 2., the $n \neq 0$ fermion KK-modes are all much heavier 

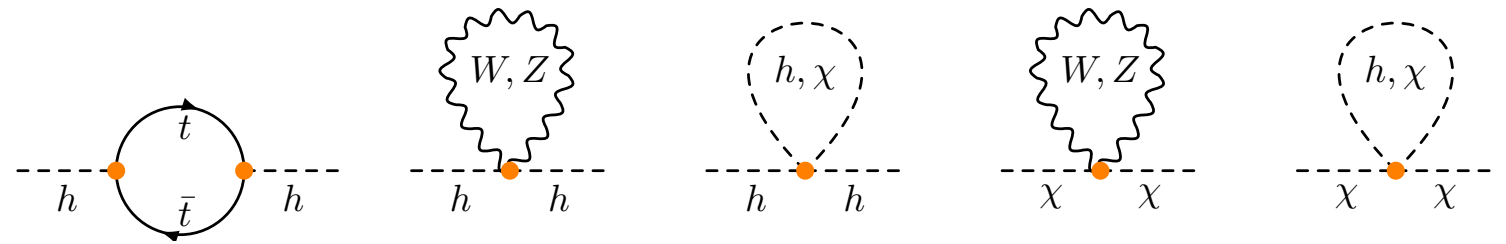

Figure 4. One-loop diagrams in the unitary gauge contributing to the Higgs boson mass and the DM scalar mass.

than the KK cutoff, $m_{K K}$, and will not significantly influence the radiative corrections to the SM Higgs mass. We leave the study of the complete fermionic sector associated with our geometric setup for future studies.

The quantum corrections to the Higgs boson $(h)$ mass and the dark-Higgs $(\chi)$ mass within our effective theory below the KK-scale are quite essential for breaking the mass degeneracy of eq. (3.41). For instance, at the 1-loop level of the perturbative expansion, the main contributions (quadratically divergent) to the masses of the SM Higgs and the dark-Higgs come from the exchanges of the top quark $(t)$, massive gauge bosons $(W, Z)$, Higgs boson $(h)$ and the dark-Higgs $(\chi)$ in the loop, ${ }^{8}$ see figure 4 . It is instructive to write the general 1-loop effective scalar potential $V_{\text {eff }}\left(H_{1}, H_{2}\right)$ for our effective theory, described in the previous section, $\mathrm{as}^{9}$

$$
V_{\text {eff }}\left(H_{1}, H_{2}\right)=V_{0}\left(H_{1}, H_{2}\right)+V_{1}\left(H_{1}, H_{2}\right),
$$

where $V_{0}\left(H_{1}, H_{2}\right)$ is the tree level scalar potential given by eq. (3.35) and $V_{1}\left(H_{1}, H_{2}\right)$ is the 1-loop effective potential, given by (see for example refs. [48-50])

$$
V_{1}\left(H_{1}, H_{2}\right)=\frac{\Lambda^{2}}{32 \pi^{2}}\left[3\left(g_{4}^{2}+\frac{1}{2}\left(g_{4}^{2}+g_{4}^{\prime 2}\right)+8 \lambda\right)\left(\left|H_{1}\right|^{2}+\left|H_{2}\right|^{2}\right)-12 y_{t}^{2}\left|H_{1}\right|^{2}\right]+\cdots,
$$

where $y_{t}$ is the top Yukawa coupling, related to top mass through $m_{t}^{2}=y_{t}^{2} v^{2} / 2$. We use the momentum cut-off regularization. Also it is important to comment here that $\mathrm{H}_{2}$ is odd under the geometric $\mathbb{Z}_{2}$ parity, implying that it does not couple to the even zero-mode fermions. Moreover, we consider only the quadratically divergent part of the effective scalar potential and the ellipses in the above equation represent the terms which are not quadratically divergent.

The minimization of the effective potential $V_{\text {eff }}\left(H_{1}, H_{2}\right)$, i.e.

$$
\left.\frac{\partial V_{\mathrm{eff}}}{\partial H_{i}}\right|_{H_{i}=\left\langle H_{i}\right\rangle}=0, \quad \text { where } \quad\left\langle H_{i}\right\rangle=\frac{1}{\sqrt{2}}\left(\begin{array}{c}
0 \\
v_{i}
\end{array}\right) \quad i=1,2
$$

\footnotetext{
${ }^{8}$ Another scalar which could be potentially present in our effective theory is the radion, which is responsible for the stabilization of the setup. The stabilization mechanism is beyond the scope of the present work, as here we assume a rigid geometrical background with no fluctuations of the 5D metric. However, we want to comment here that if the radion were present in our effective theory, because of its bosonic nature it would likely reduce the fine-tuning much in the manner that the $\chi$ does.

${ }^{9}$ Note that in this section we are considering the Higgs doublets $H_{1,2}$ in the unitary gauge, such that $H_{1}(x)=\frac{1}{\sqrt{2}}\left(\begin{array}{c}0 \\ v_{1}+h\end{array}\right)$ and $H_{2}(x)=\frac{1}{\sqrt{2}}\left(\begin{array}{c}0 \\ v_{2}+\chi\end{array}\right)$, where at tree level our choice was $v_{1}=v$ and $v_{2}=0$.
} 
gives the following set of conditions for the global minimum,

$$
\lambda v_{1}^{2}=\mu^{2}-\delta \mu^{2}-3 \lambda v_{2}^{2}, \quad \text { or } \quad v_{1}=0,
$$

and

$$
\lambda v_{2}^{2}=\mu^{2}-\delta \mu^{2}+\frac{3}{8} \frac{\Lambda^{2}}{\pi^{2}} y_{t}^{2}-3 \lambda v_{1}^{2}, \quad \text { or } \quad v_{2}=0,
$$

where $\delta \mu^{2}$ is given by

$$
\delta \mu^{2}=\frac{3 \Lambda^{2}}{32 \pi^{2}}\left[g_{4}^{2}+\frac{1}{2}\left(g_{4}^{2}+g_{4}^{\prime 2}\right)+8 \lambda-4 y_{t}^{2}\right] .
$$

Of the four possible global minima of eqs. (3.46) and (3.47), we will choose the vacuum such that $H_{1}$ acquires the vev, whereas $H_{2}$ does not:

$$
v_{1}=v, \quad v_{2}=0,
$$

where $v \simeq 246 \mathrm{GeV}$ is the vacuum expectation value of the SM Higgs doublet. With this choice of vacuum, the 1-loop corrected masses for the fluctuations around the vacuum are

$$
\begin{aligned}
m_{h}^{2}=\left.\frac{\partial^{2} V_{\mathrm{eff}}\left(H_{1}, H_{2}\right)}{\partial H_{1}^{2}}\right|_{H_{1}=v, H_{2}=0} & =\left(-\mu^{2}+\delta \mu^{2}\right)+3 \lambda v^{2}=2 \lambda v^{2}, \\
m_{\chi}^{2}=\left.\frac{\partial^{2} V_{\mathrm{eff}}\left(H_{1}, H_{2}\right)}{\partial H_{2}^{2}}\right|_{H_{1}=v, H_{2}=0} & =\left(-\mu^{2}+\delta \mu^{2}\right)+3 \lambda v^{2}+\frac{3}{8} \frac{\Lambda^{2}}{\pi^{2}} y_{t}^{2}, \\
& =2 \lambda v^{2}+\frac{3}{4} \frac{\Lambda^{2}}{\pi^{2} v^{2}} m_{t}^{2} .
\end{aligned}
$$

To get $m_{h}=125 \mathrm{GeV}$, equivalent to $v \simeq 246 \mathrm{GeV}$, we need to fine-tune the parameters of the theory. To quantify the level of fine-tuning, we employ the Barbieri-Giudice fine-tuning measure $\Delta_{m_{h}}[49-51]$ :

$$
\Delta_{m_{h}} \equiv\left|\frac{\delta \mu^{2}}{\mu^{2}}\right|=\left|\frac{\delta m_{h}^{2}}{m_{h}^{2}}\right| .
$$

We plot the fine-tuning measure $\Delta_{m_{h}}$ as a function of the effective cutoff scale $\Lambda$ in figure 5 . If one allows fine-tuning of about $10 \%$, i.e. $\Delta_{m_{h}}=10$, then the effective cutoff scale is $\Lambda \simeq 2 \mathrm{TeV}$. The most stringent bounds on the KK-scale $m_{K K}$ in RS1 geometry with a bulk Higgs come from electroweak precision tests (EWPT) by fitting the $S, T$ and $U$ parameters [26]. The lower bound on the KK mass scale in our model (AdS geometry, i.e. $A(y)=-k|y|)$ is $m_{K K} \gtrsim 2.5 \mathrm{TeV}$ for $\beta=0$ and $m_{K K} \gtrsim 4.3 \mathrm{TeV}$ for $\beta=10$ at 95\% C.L. [26]. This implies a tension between fine-tuning (naturalness) and the lower bound on the KK mass scale $m_{K K}$. The region within the gray lines in figure 5 shows the current bounds on the KK mass scale for our geometry and the associated fine-tuning. It is important to comment here that a slight modification to the AdS geometry (for example, models with soft wall or thick branes) leads to a considerable relaxation of the above mentioned lower bound on KK mass scale [18-20]. For instance, a mild modification to the AdS metric in the vicinity of the IR-brane can relax the KK mass scale to $m_{K K} \gtrsim$ $1 \mathrm{TeV}$ [18-20, 29, 52]. Needless to say, the generalization of our model to modified AdS geometries with soft walls or thick branes is possible. 

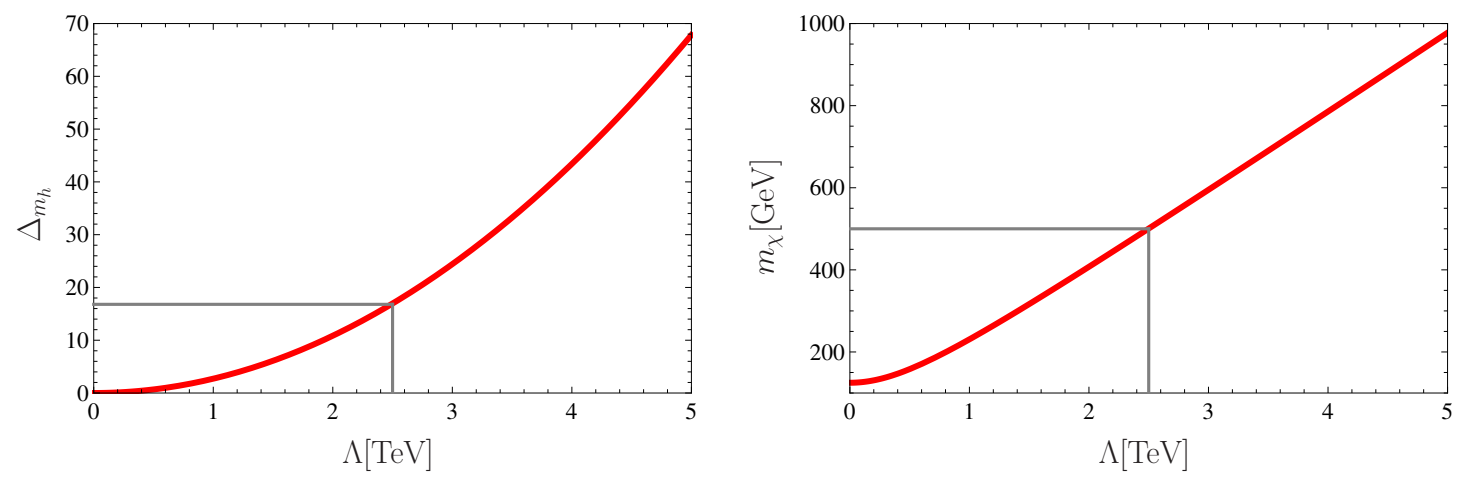

Figure 5. The left plot gives the value of the fine-tuning measure $\Delta_{m_{h}}$ for a Higgs mass of $125 \mathrm{GeV}$ as a function of the cutoff $\Lambda$. The right plot shows the dependence of $m_{\chi}$ on $\Lambda$ for $m_{h}=125 \mathrm{GeV}$. In our model $\Lambda=m_{K K}$. The vertical gray line indicates the current lower bound on the KK mass scale coming from EWPT as computed in our model for $\beta=0, m_{K K} \gtrsim 2.5 \mathrm{TeV}$.

The 1-loop quantum corrected dark matter squared mass $m_{\chi}^{2}$ is:

$$
m_{\chi}^{2}=m_{h}^{2}+\frac{3}{4} \frac{\Lambda^{2}}{\pi^{2} v^{2}} m_{t}^{2}
$$

Hence, $m_{\chi}$ is raised linearly with the cut-off scale $\Lambda$. This is illustrated in figure 5 . An interesting aspect of our model is that dark matter is predicted to be heavier than the SM Higgs boson. A natural value of the cutoff coincides with the mass of the first KK excitations, which are experimentally limited [53] to lie above a few $\mathrm{TeV}$ (depending on model details and KK mode sought). Requiring that the fine-tuning measure $\Delta_{m_{h}}$ be less than 100 implies that $m_{K K}$ should be below about $6 \mathrm{TeV}$. Meanwhile, the strongest version of the EWPT bound requires $m_{K K} \gtrsim 2.5 \mathrm{TeV}$, corresponding to $m_{\chi} \sim 500 \mathrm{GeV}$, for which $\Delta_{m_{h}}$ is a very modest $\sim 18$. In short, our model is most consistent for $500 \mathrm{GeV} \lesssim m_{\chi} \lesssim$ $1200 \mathrm{GeV}$.

\subsection{Dark matter relic abundance}

In this subsection we calculate the dark matter relic abundance. The diagrams contributing to dark matter annihilation are shown in figure 6 . The squared amplitudes $|\mathcal{M}|^{2}$ corresponding to the contribution of each final state to dark matter annihilation are:

$$
\begin{aligned}
|\mathcal{M}(\chi \chi \rightarrow \tilde{V} \tilde{V})|^{2} & =\frac{4 m_{\tilde{V}}^{4}}{S_{\tilde{V}} v^{4}}\left(1+\frac{3 m_{h}^{2}}{s-m_{h}^{2}}\right)^{2}\left[2+\left(1-\frac{s}{2 m_{\tilde{V}}^{2}}\right)^{2}\right] \\
|\mathcal{M}(\chi \chi \rightarrow f \bar{f})|^{2} & =18 N_{c} \frac{m_{f}^{2} m_{h}^{4}}{v^{4}} \frac{s-4 m_{f}^{2}}{\left(s-m_{h}^{2}\right)^{2}} \\
|\mathcal{M}(\chi \chi \rightarrow h h)|^{2} & =\frac{9 m_{h}^{4}}{2 v^{4}}\left[1+3 m_{h}^{2}\left(\frac{1}{s-m_{h}^{2}}+\frac{1}{t-m_{\chi}^{2}}+\frac{1}{u-m_{\chi}^{2}}\right)\right]^{2}
\end{aligned}
$$

where $\tilde{V}=W, Z$ and $S_{W}=1$ and $S_{Z}=2$ are the symmetry factors accounting for the identical particles in the final state; $N_{c}$ refers to the number of "color" degrees of freedom 

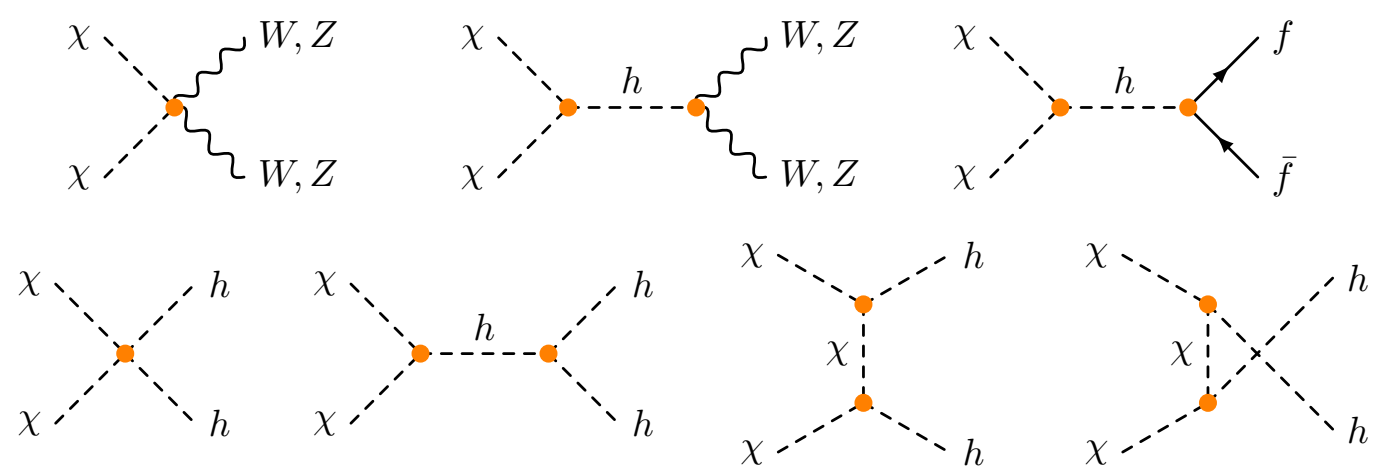

Figure 6. Dark matter annihilation diagrams.
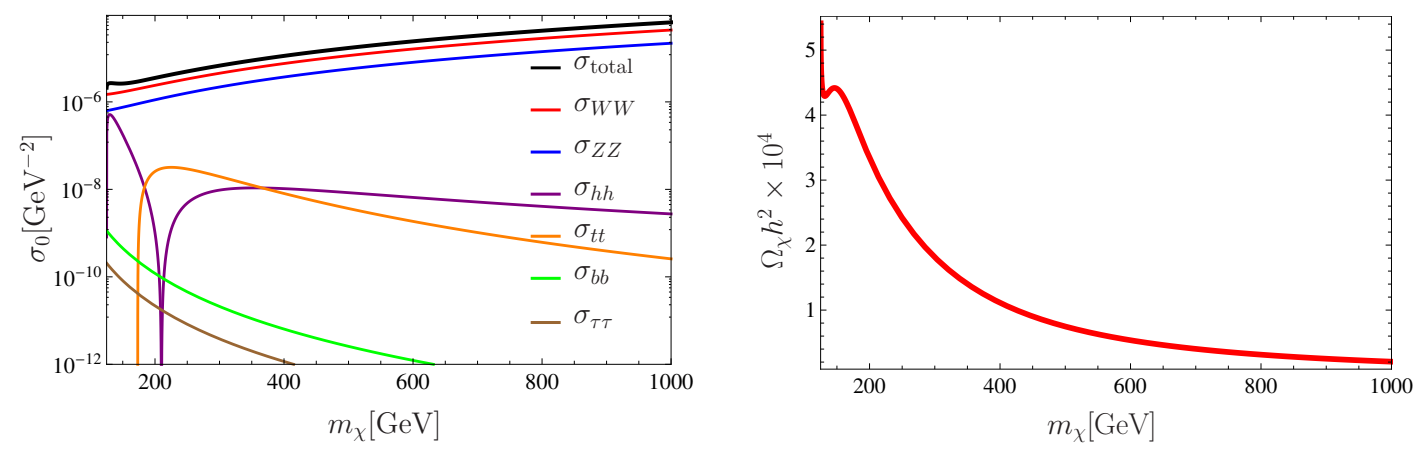

Figure 7. The above graphs show the annihilation cross-section $\sigma_{0}$ for different final states (left) and the $\chi$ abundance $\Omega_{\chi} h^{2} \times 10^{4}$ (right) as a function of dark matter mass $m_{\chi}$.

for the given fermion and $s, t, u$ are the Mandelstam variables. Here, we ignore the loopinduced $\gamma \gamma$ and $Z \gamma$ final states, which are strongly suppressed. Note that the first term in the parenthesis in eq. (3.53) and the first term in the square bracket in eq. (3.55) arise from the $\chi \chi \tilde{V} \tilde{V}$ and the $\chi \chi h h$ contact interactions, respectively. The former channel is present in our model since $\chi$ is a component of the (truncated) odd $\mathrm{SU}(2)$ doublet.

In the left panel of figure 7 we plot the annihilation cross-section for the contributing channels as a function of $m_{\chi}$. (Note that the parameter $\Lambda$ would only enter if we performed this calculation at the one-loop level.) As seen from the plot, the total cross section is dominated by $W W$ and $Z Z$ final states. The main contributions for these final states are those generated by the contact interactions $\chi \chi \tilde{V} \tilde{V}$. In fact, in our model, the $\tilde{V} \tilde{V}$ final states are additionally enhanced by a constructive interference of the contact $\chi \chi \tilde{V} \tilde{V}$ interaction with the s-channel Higgs-exchange diagram. In addition, for low $m_{\chi}$, there is a comparable contribution from $\chi \chi$ annihilation into $h h$. (The dip at $m_{\chi} \sim 210 \mathrm{GeV}$ is caused by cancellation between the contact interaction and $s, t, u$-channel diagrams.) Fermionic final states are always irrelevant; even $\chi \chi \rightarrow t \bar{t}$ production is very small in comparison to $\chi \chi \rightarrow \tilde{V} \tilde{V}$. Then, adopting the standard s-wave cold dark matter approximation [54], we find the present $\chi$ abundance $\Omega_{\chi} h^{2}$ shown in the right panel of figure 7 . We observe that $\Omega_{\chi} h^{2} \lesssim 10^{-4}$ once the EWPT bound of $m_{\chi} \gtrsim 500 \mathrm{GeV}$ is imposed. Clearly, some other dark matter component is needed within this model to satisfy the Planck measurement, $\Omega_{\chi} h^{2} \sim 0.1[32]$. 


\section{Summary}

In this paper, we constructed a model with $\mathbb{Z}_{2}$ geometric symmetry such that two identical AdS patches are glued together at $y=0$, where $y$ is the coordinate of the fifth dimension. We considered three D3-branes, one at $y=0$ referred to as the UV-brane where gravity is assumed to be localized and two branes at $y= \pm L$ referred to as IR-branes - the IR-UVIR model. For this $\mathbb{Z}_{2}$ symmetric geometric setup we found that the RS metric (1.1) is the background solution of pure gravity when the matter backreaction is neglected. To investigate possible backreaction of matter fields (bulk SU(2) Higgs doublet) on the geometry, we solved the $5 \mathrm{D}$ coupled scalar-gravity equations of motion adopting the superpotential method. We found analytic solutions where the Higgs background vev is highly localized towards the IR brane. It was also verified that the backreaction is negligible for $k L \simeq 37$ which is required in order to address the gauge hierarchy problem. The technique employed to find solutions is very general and can be used to any bulk Higgs RS1-like constructions to stabilize geometry taking into account backreaction.

The motivation of this work is twofold: $(i)$ to analyze the situation where EWSB is due to the bulk Higgs in this $\mathbb{Z}_{2}$ symmetric geometry; and (ii) to discuss the lowest odd KK-mode as a dark matter candidate. Concerning EWSB, we discussed in detail many important aspects of SSB due to a bulk Higgs. In the appendix A we considered the Abelian gauge group where we discussed two alternative approaches to SSB due to a bulk Higgs field acquiring a vacuum expectation value. In one approach, the symmetry breaking is triggered by a vev of the KK zero-mode of the bulk Higgs field. The second approach is based on the expansion of the bulk Higgs field around an extra-dimensional vev with non-trivial $y$ profile. The comparison between the two Abelian scenarios is summarized in tables 1 and 2. The (zero-mode) effective theories obtained from the two approaches are identical and the most intriguing feature of the Abelian Higgs mechanism is that the even and odd Higgs zero-modes have degenerate mass at the tree-level - a feature that is also present in the SM case.

To achieve SSB, the choice of boundary conditions for the fields at $\pm L$ is critical. In both approaches to the Abelian case, we allowed the $y$-derivative of a field to have an arbitrary value at $\pm L$ as opposed to requiring that the field value itself be zero, i.e. we employed Neumann or mixed b.c. rather than Dirichlet b.c. at $\pm L$. The latter choice would have led to an explicit symmetry breaking scenario in which there are no Goldstone modes and the gauge bosons do not acquire mass. (Note that the boundary or "jump" conditions at $y=0$ follow from the bulk equations of motion in the case of even modes, whereas odd modes are required to be zero by symmetry.)

Following this introductory material in appendix A, we considered EWSB assuming that the SM gauge group is present in the bulk of our $\mathbb{Z}_{2}$ symmetric $5 \mathrm{D}$ warped model. The zero-mode effective theory appropriate at scales below the KK scale, $m_{K K}$, was obtained. For appropriate Higgs field potentials in the bulk and localized at the UV and IR-branes, SM-like EWSB is obtained when only the IR-branes have a quartic potential term. In contrast, quadratic mass-squared terms are allowed both on the branes and in the bulk. 
Of course, to achieve spontaneous EWSB, we employed the same boundary conditions as delineated above for the Abelian model. The resulting model has the following features.

- Due to warped KK-parity all fields develop even and odd towers of KK-modes in the $4 \mathrm{D}$ effective theory.

- Assuming that the KK-scale is high enough $\left(m_{K K} \sim \mathcal{O}(\right.$ few $\left.) \mathrm{TeV}\right)$, we derive the low energy effective theory which includes only zero-modes of the theory.

- In the effective theory, the symmetry of the model is $\left[\mathrm{SU}(2) \times \mathrm{U}(1)_{Y}\right]^{\prime} \times[\mathrm{SU}(2) \times$ $\left.\mathrm{U}(1)_{Y}\right]$, where the unprimed symmetry group is gauged while the other stays as a global symmetry. The zero-mode odd gauge fields and the corresponding Goldstone modes from the odd Higgs doublet are eliminated due to the b.c..

- In the low energy effective theory, we are left with all the SM fields plus a dark-Higgs — the odd zero-mode Higgs. This dark-Higgs and the SM Higgs (the even zero-mode) are degenerate at tree level.

- In order to get the SM Higgs mass of $125 \mathrm{GeV}$, we need to fine-tune the 5D fundamental parameters of theory to about $1 \%-5 \%$, where the upper bound is determined by the lower bound on the KK scale coming from EWPT requirements.

- We computed the one-loop quantum corrections to the tree-level masses of the SM Higgs and the dark Higgs assuming that the cutoff scale of our effective theory is the KK-scale, $m_{K K}$. One finds that the dark-Higgs mass is necessarily larger than the SM Higgs mass, the difference being quadratically dependent on $m_{K K}$.

- Requiring that the fine-tuning measure $\Delta_{m_{h}}$ be less than 100 implies that $m_{K K}$ should be below about $6 \mathrm{TeV}$. Meanwhile, the strongest version of the EWPT bound requires $m_{K K} \gtrsim 2.5 \mathrm{TeV}$, corresponding to $m_{\chi} \sim 500 \mathrm{GeV}$, for which $\Delta_{m_{h}}$ is a very modest $\sim 18$. In short, our model is most consistent for $500 \mathrm{GeV} \lesssim m_{\chi} \lesssim 1200 \mathrm{GeV}$.

- We calculate the relic abundance of the dark-Higgs in the cold dark matter approximation. For $m_{\chi}$ in the above preferred range, $\Omega_{\chi} h^{2} \lesssim 10^{-4}$ as compared to the current experimental value of $\sim 0.1$. To obtain a more consistent dark matter density, one needs to either assume another DM particle or perform a more rigorous analysis of our model by considering the even and odd higher KK-modes in the effective theory.

\section{Acknowledgments}

JFG and YJ are supported in part by US DOE grant DE-SC-000999. AA and BG acknowledge partial support by the National Science Centre (Poland) research project, decision no DEC-2014/13/B/ST2/03969. The work of AA was partially supported by the Foundation for Polish Science International PhD Projects Programme co-financed by the EU European Regional Development Fund. AA and BG are grateful to the Mainz Institute for Theoretical Physics (MITP) and the University of California Davis for their hospitality and partial support during the completion of this work. JFG and YJ acknowledge hospitality and partial support by Warsaw University during the course of the project. AA would like to thank Adam Falkowski, Matthias Neubert and Mariano Quiros for useful discussions. 


\section{A SSB in the IR-UV-IR model: the Abelian Higgs mechanism}

In this appendix we will discuss the mechanism of spontaneous symmetry breaking (SSB) for an Abelian case with the Higgs field (a complex scalar) in the IR-UV-IR geometry of section 2.1. The metric is given by eq. (1.1), we will neglect the back reaction of the bulk fields on the geometry. We start by specifying the 5D Abelian action,

$$
\begin{aligned}
S_{A b}=-\int d^{5} x \sqrt{-g}\{ & \frac{1}{4} F_{M N} F^{M N}+\left(D_{M} \Phi\right)^{*} D^{M} \Phi+\mu_{B}^{2} \Phi^{*} \Phi \\
& \left.+V_{\mathrm{IR}}(\Phi) \delta(y+L)+V_{\mathrm{UV}}(\Phi) \delta(y)+V_{\mathrm{IR}}(\Phi) \delta(y-L)\right\},
\end{aligned}
$$

where $D_{M} \equiv \partial_{M}-i g_{5} A_{M}$ with the $5 \mathrm{D} \mathrm{U}(1)$ coupling constant $g_{5}$ and $F_{M N} \equiv \partial_{M} A_{N}-$ $\partial_{N} A_{M}$. We require that the bulk potential and the UV-brane potential have only quadratic terms whereas the IR-brane potential is allowed to have a quartic term:

$$
V_{\mathrm{UV}}(\Phi)=\frac{m_{\mathrm{UV}}^{2}}{k} \Phi^{*} \Phi, \quad \quad V_{\mathrm{IR}}(\Phi)=-\frac{m_{\mathrm{IR}}^{2}}{k} \Phi^{*} \Phi+\frac{\lambda_{\mathrm{IR}}}{k^{2}}\left(\Phi^{*} \Phi\right)^{2} .
$$

In this way EWSB is mainly triggered by the IR-brane. Above, $\Phi$ is a complex scalar field and the parametrization is such that $m_{\mathrm{UV}}$ and $m_{\mathrm{IR}}$ have mass dimensions while $\lambda_{\mathrm{IR}}$ is dimensionless. The gauge transformations can be written as

$$
\begin{aligned}
\Phi(x, y) & \rightarrow \Phi^{\prime}(x, y)=e^{i \Lambda(x, y)} \Phi(x, y), \\
A_{M}(x, y) & \rightarrow A_{M}^{\prime}(x, y)=A_{M}(x, y)+\frac{1}{g_{5}} \partial_{M} \Lambda(x, y),
\end{aligned}
$$

where $\Lambda(x, y)$ is the gauge parameter.

As discussed in section 2.3, the fields in the IR-UV-IR setup have even and odd bulk wave functions implied by the geometric KK-parity. Hence, it is convenient to decompose the generic Higgs and the gauge field into fields of definite parity as follows

$$
\Phi(x, y)=\Phi^{(+)}(x, y)+\Phi^{(-)}(x, y), \quad A_{M}(x, y)=A_{M}^{(+)}(x, y)+A_{M}^{(-)}(x, y),
$$

where \pm denotes the even and odd states. The gauge transformations for the even and odd parity modes are,

$$
\begin{gathered}
A_{\mu}^{( \pm)}(x, y) \rightarrow A_{\mu}^{\prime( \pm)}(x, y)=A_{\mu}^{( \pm)}(x, y)+\frac{1}{g_{5}} \partial_{\mu} \Lambda^{( \pm)}(x, y) \\
A_{5}^{( \pm)}(x, y) \rightarrow A_{5}^{\prime( \pm)}(x, y)=A_{5}^{( \pm)}(x, y)+\frac{1}{g_{5}} \partial_{5} \Lambda^{(\mp)}(x, y) . \\
\left(\begin{array}{l}
\Phi^{(+)}(x, y) \\
\Phi^{(-)}(x, y)
\end{array}\right) \rightarrow\left(\begin{array}{l}
\Phi^{\prime(+)}(x, y) \\
\Phi^{\prime(-)}(x, y)
\end{array}\right)=e^{i \Lambda^{(+)}(x, y) \mathbb{1}} e^{i \Lambda^{(-)}(x, y) \tau_{1}}\left(\begin{array}{l}
\Phi^{(+)}(x, y) \\
\Phi^{(-)}(x, y)
\end{array}\right),
\end{gathered}
$$

where $\mathbb{1}$ is a $2 \times 2$ unit matrix, whereas $\tau_{1}$ is the first Pauli matrix.

With this decomposition the above action can be written as

$$
S_{A b}=-\int d^{5} x \sqrt{-g}\left\{\frac{1}{4} F_{\mu \nu}^{(+)} F_{(+)}^{\mu \nu}+\frac{1}{2} F_{\mu 5}^{(+)} F_{(+)}^{\mu 5}+D_{M} \Phi^{(+) *} D^{M} \Phi^{(+)}+\mu_{B}^{2} \Phi^{(+) *} \Phi^{(+)}\right.
$$




$$
\begin{aligned}
& +\frac{1}{4} F_{\mu \nu}^{(-)} F_{(-)}^{\mu \nu}+\frac{1}{2} F_{\mu 5}^{(-)} F_{(-)}^{\mu 5}+D_{M} \Phi^{(-) *} D^{M} \Phi^{(-)}+\mu_{B}^{2} \Phi^{(-) *} \Phi^{(-)} \\
& \left.+V_{\mathrm{IR}}\left(\Phi^{( \pm)}\right) \delta(y+L)+V_{\mathrm{UV}}\left(\Phi^{(+)}\right) \delta(y)+V_{\mathrm{IR}}\left(\Phi^{( \pm)}\right) \delta(y-L)\right\}
\end{aligned}
$$

where,

$$
F_{\mu \nu}^{( \pm)} \equiv \partial_{\mu} A_{\nu}^{( \pm)}-\partial_{\mu} A_{\nu}^{( \pm)}, \quad F_{\mu 5}^{( \pm)} \equiv \partial_{\mu} A_{5}^{( \pm)}-\partial_{5} A_{\mu}^{(\mp)}
$$

The brane localized potentials for the Higgs field, $V_{\mathrm{UV}}(\Phi)$ and $V_{\mathrm{IR}}(\Phi)$, can be written in terms of even and odd parity modes as

$$
\begin{aligned}
V_{\mathrm{UV}}\left(\Phi^{(+)}\right)= & \frac{m_{\mathrm{UV}}^{2}}{k}\left|\Phi^{(+)}\right|^{2}, \\
V_{\mathrm{IR}}\left(\Phi^{( \pm)}\right)= & -\frac{m_{\mathrm{IR}}^{2}}{k}\left|\Phi^{(+)}\right|^{2}-\frac{m_{\mathrm{IR}}^{2}}{k}\left|\Phi^{(-)}\right|^{2}+\frac{\lambda_{\mathrm{IR}}}{k^{2}}\left|\Phi^{(+)}\right|^{4}+\frac{\lambda_{\mathrm{IR}}}{k^{2}}\left|\Phi^{(-)}\right|^{4} \\
& +\frac{4 \lambda_{\mathrm{IR}}}{k^{2}}\left|\Phi^{(+)}\right|^{2}\left|\Phi^{(-)}\right|^{2}+\frac{\lambda_{\mathrm{IR}}}{k^{2}}\left(\left(\Phi^{(+) *} \Phi^{(-)}\right)^{2}+\text { h.c. }\right) .
\end{aligned}
$$

Above, we have not written $\Phi^{(-)}$terms in $V_{\mathrm{UV}}$ since $\Phi^{(-)}(0)=0$. Moreover, we have not written terms in the above action, including the potentials, which are explicitly odd as they will not contribute after integration over the $y$-coordinate. One can easily check that the above brane potentials are invariant under the gauge transformations defined above. Also note that $F_{\mu \nu}^{( \pm)}$and $F_{\mu 5}^{( \pm)}$are gauge invariant under the gauge transformations (A.6) and (A.7). In the even/odd basis, the covariant derivatives $D_{\mu}$ and $D_{5}$ following from $D_{M} \equiv \partial_{M}-i g_{5} A_{M}$, take the form

$$
\begin{aligned}
& D_{\mu}\left(\begin{array}{l}
\Phi^{(+)} \\
\Phi^{(-)}
\end{array}\right) \equiv\left[\partial_{\mu}-i g_{5}\left(\begin{array}{ll}
A_{\mu}^{(+)} & A_{\mu}^{(-)} \\
A_{\mu}^{(-)} & A_{\mu}^{(+)}
\end{array}\right)\right]\left(\begin{array}{l}
\Phi^{(+)} \\
\Phi^{(-)}
\end{array}\right), \\
& D_{5}\left(\begin{array}{l}
\Phi^{(+)} \\
\Phi^{(-)}
\end{array}\right) \equiv\left[\partial_{5}-i g_{5}\left(\begin{array}{ll}
A_{5}^{(-)} & A_{5}^{(+)} \\
A_{5}^{(+)} & A_{5}^{(-)}
\end{array}\right)\right]\left(\begin{array}{l}
\Phi^{(+)} \\
\Phi^{(-)}
\end{array}\right) .
\end{aligned}
$$

It is important to note that the above action is manifestly gauge invariant under the gauge group $\mathrm{U}(1)^{\prime} \times \mathrm{U}(1)$.

The next two sub-appendices are devoted to two possible strategies for implementing spontaneous gauge symmetry breaking for this Abelian $\mathrm{U}(1)$ symmetric case. We are going to describe and compare: (i) SSB by vacuum expectation values of the KK modes and (ii) SSB by a $y$-dependent vacuum expectation value of the 5D Higgs field.

\section{A.1 SSB by vacuum expectation values of KK modes}

In this case we will choose the $5 \mathrm{D}$ axial gauge, $A_{5}^{( \pm)}=0$. This gauge is realized by choosing the gauge parameter such that,

$$
\Lambda^{( \pm)}(x, y)=-g_{5} \int d y A_{5}^{(\mp)}(x, y)+\hat{\Lambda}^{( \pm)}(x)
$$

where $\hat{\Lambda}^{( \pm)}(x)$ is the integration constant (residual gauge freedom) and only depends on $x^{\mu}$. Note that the $\hat{\Lambda}^{(-)}(x)$, being an odd function of $y$, must vanish. Consequently, we are left with only one $4 \mathrm{D}$ gauge function, $\hat{\Lambda}^{(+)}(x)$. 
It is convenient to parameterize the complex scalar field $\Phi^{( \pm)}(x, y)$ as

$$
\left(\begin{array}{c}
\Phi^{(+)} \\
\Phi^{(-)}
\end{array}\right) \equiv e^{i g_{5}\left(\pi^{(+)} \mathbb{1}+\pi^{(-)} \tau_{1}\right)}\left(\begin{array}{c}
\phi^{(+)} \\
\phi^{(-)}
\end{array}\right)
$$

where $\phi^{( \pm)}(x, y)$ and $\pi^{( \pm)}(x, y)$ are real scalar fields. We KK-decompose the scalar fields $\phi^{( \pm)}(x, y), \pi^{( \pm)}(x, y)$ and the gauge fields $A_{\mu}^{( \pm)}(x, y)$ as

$$
\begin{array}{cc}
\phi^{( \pm)}(x, y) & =\sum_{n} \phi_{n}^{( \pm)}(x) f_{n}^{( \pm)}(y) \\
\pi^{( \pm)}(x, y)=\sum_{n} \pi_{n}^{( \pm)}(x) a_{n}^{( \pm)}(y), & A_{\mu}^{( \pm)}(x, y)=\sum_{n} A_{\mu n}^{( \pm)}(x) a_{n}^{( \pm)}(y)
\end{array}
$$

where the scalar wave-functions $f_{n}^{( \pm)}(y)$ and the gauge wave-functions $a_{n}^{( \pm)}(y)$ satisfy

$$
\begin{aligned}
-\partial_{5}\left(e^{4 A(y)} \partial_{5} f_{n}^{( \pm)}(y)\right)+\mu_{B}^{2} e^{4 A(y)} f_{n}^{( \pm)}(y) & =m_{n}^{( \pm) 2} e^{2 A(y)} f_{n}^{( \pm)}(y) \\
-\partial_{5}\left(e^{2 A(y)} \partial_{5} a_{n}^{( \pm)}(y)\right) & =m_{A_{n}^{( \pm)}}^{2} a_{n}^{( \pm)}(y) .
\end{aligned}
$$

The wave-functions $f_{n}^{( \pm)}$and $a_{n}^{( \pm)}$satisfy the following orthonormality conditions,

$$
\int_{-L}^{+L} d y e^{2 A(y)} f_{m}^{( \pm)}(y) f_{n}^{( \pm)}(y)=\delta_{m n}, \quad \int_{-L}^{+L} d y a_{m}^{( \pm)}(y) a_{n}^{( \pm)}(y)=\delta_{m n}
$$

It is worth commenting here that the gauge field $A_{\mu}^{( \pm)}(x, y)$ and the scalar field $\pi^{( \pm)}(x, y)$ share the same $y$-dependent KK-eigen bases $a_{n}^{( \pm)}(y)$, as is necessitated (see below) by the Higgs mechanism. The KK-modes satisfy $\square^{(4)} A_{\mu n}^{( \pm)}(x)=m_{A_{n}^{( \pm)}}^{2} A_{\mu n}^{( \pm)}(x)$. The boundary (jump) conditions for $a_{n}^{( \pm)}(y)$ at $y=0$ and $y= \pm L$ are,

$$
\left.\partial_{5} a_{n}^{(+)}(y)\right|_{0^{+}}=0,\left.\quad a_{n}^{(-)}(y)\right|_{0^{+}}=0,\left.\quad \partial_{5} a_{n}^{( \pm)}(y)\right|_{ \pm L^{\mp}}=0
$$

We choose the Neumann b.c. for $a_{n}^{(+)}(y)$ at $y=0, \pm L$ in order to insure that we get non-zero even zero-mode gauge profiles. With regard to the odd modes, we have chosen the Neumann b.c. of $\partial_{5} a_{n}^{(-)}( \pm L)=0$ - the other choice of $a_{n}^{(-)}( \pm L)=0$ would lead to a trivial theory with $a_{n}^{(-)}(y)=0$ everywhere. Similarly, the jump and boundary conditions for the even and odd scalar profiles $f_{n}^{(+)}(y)$ are,

$$
\left.\left(\partial_{5}-\frac{m_{\mathrm{UV}}^{2}}{2 k}\right) f_{n}^{(+)}(y)\right|_{0}=0,\left.\quad f_{n}^{(-)}(y)\right|_{0}=0,\left.\quad\left( \pm \partial_{5}-\frac{m_{\mathrm{IR}}^{2}}{k}\right) f_{n}^{( \pm)}(y)\right|_{ \pm L}=0
$$

Assuming that the KK-scale is high enough, i.e. $m_{K K} \sim \mathcal{O}$ (few) $\mathrm{TeV}$, we can employ the effective theory where only the lowest modes (zero-modes with masses much below $m_{K K}$ ) are considered. Equation (A.20) along with the b.c. (A.22) imply that the odd zero-mode wave-function of the gauge boson is zero, i.e. $a_{0}^{(-)}=0$. As a result, in the effective theory the odd zero-mode gauge boson $A_{0 \mu}^{(-)}$and odd parity Goldstone mode $\pi_{0}^{(-)}$are not present. In contrast, the even zero-mode wave-function for the gauge boson has a constant profile 
in the bulk, i.e. $a_{0}^{(+)}=1 / \sqrt{2 L}$. The forms of the scalar zero-mode wave functions $f_{0}^{( \pm)}(y)$ are given by:

$$
f_{0}^{( \pm)}(|y|) \simeq \sqrt{k(1+\beta)} e^{k L} e^{(2+\beta) k(|y|-L)}
$$

where, $f_{0}^{(-)}(y)=\epsilon(y) f_{0}^{(-)}(|y|)$. The low-energy effective action for the zero-modes is

$$
\begin{aligned}
S_{A b}^{\mathrm{eff}}=-\int d^{4} x & \left\{\frac{1}{4} F_{\mu \nu}^{0(+)} F_{0(+)}^{\mu \nu}+\partial_{\mu} \phi_{0}^{(+)} \partial^{\mu} \phi_{0}^{(+)}-\mu^{2} \phi_{0}^{(+) 2}+\partial_{\mu} \phi_{0}^{(-)} \partial^{\mu} \phi_{0}^{(-)}-\mu^{2} \phi_{0}^{(-) 2}\right. \\
& +\left(g_{0000}^{(+) 2} \phi_{0}^{(+) 2}+\bar{g}_{0000}^{(+) 2} \phi_{0}^{(-) 2}\right)\left(A_{0 \mu}^{(+)}-\partial_{\mu} \pi_{0}^{(+)}\right)^{2} \\
& \left.+\lambda_{0000}^{(+)} \phi_{0}^{(+) 4}+\lambda_{0000}^{(-)} \phi_{0}^{(-) 4}+6 \lambda_{0000} \phi_{0}^{(+) 2} \phi_{0}^{(-) 2}\right\}
\end{aligned}
$$

where the mass parameter $\mu$ is defined as $\mu^{2} \equiv(1+\beta) m_{K K}^{2} \delta_{\mathrm{IR}}$, with the parameters

$$
\delta_{\mathrm{IR}} \equiv \frac{m_{\mathrm{IR}}^{2}}{k^{2}}-2(2+\beta), \quad m_{K K} \equiv k e^{-k L} \quad \text { and } \quad \beta \equiv \sqrt{4+\mu_{B}^{2} / k^{2}} .
$$

Above, the couplings have the following form in terms of the parameters of the $5 \mathrm{D}$ theory:

$$
\lambda_{0000}^{( \pm)}=\lambda_{0000} \simeq \lambda \equiv \lambda_{\mathrm{IR}}(1+\beta)^{2}, \quad g_{0000}^{(+)}=\bar{g}_{0000}^{(+)}=g_{4} \equiv \frac{g_{5}}{\sqrt{2 L}} .
$$

Our effective theory can be parameterized by redefining $A_{0 \mu}^{(+)}(x) \equiv A_{\mu}(x), \pi_{0}^{(+)}(x) \equiv \pi(x)$,

$$
\Phi_{1}(x) \equiv e^{i g_{4} \pi(x)} \phi_{0}^{(+)}(x), \quad \Phi_{2}(x) \equiv e^{i g_{4} \pi(x)} \phi_{0}^{(-)}(x),
$$

in which case the above effective action can be written in a nice gauge covariant form as

$$
S_{A b}^{\mathrm{eff}}=-\int d^{4} x\left\{\frac{1}{4} F_{\mu \nu} F^{\mu \nu}+\mathcal{D}_{\mu} \Phi_{1}^{*} \mathcal{D}^{\mu} \Phi_{1}+\mathcal{D}_{\mu} \Phi_{2}^{*} \mathcal{D}^{\mu} \Phi_{2}+V\left(\Phi_{1}, \Phi_{2}\right)\right\},
$$

where the covariant derivative is defined as $\mathcal{D}_{\mu} \equiv \partial_{\mu}-i g_{4} A_{\mu}$ and the scalar potential can be written as

$$
V\left(\Phi_{1}, \Phi_{2}\right)=-\mu^{2}\left|\Phi_{1}\right|^{2}-\mu^{2}\left|\Phi_{2}\right|^{2}+\lambda\left|\Phi_{1}\right|^{4}+\lambda\left|\Phi_{2}\right|^{4}+6 \lambda\left|\Phi_{1}\right|^{2}\left|\Phi_{2}\right|^{2} .
$$

It is important to note that, after choosing the gauge $A_{5}(x, y)=0$, we are left with a residual gauge freedom with a single purely $4 \mathrm{D}$ gauge parameter $\hat{\Lambda}^{(+)}(x)$ such that the above Lagrangian is invariant under the $\mathrm{U}(1)$ gauge transformation,

$$
\begin{gathered}
A_{\mu}(x) \rightarrow A_{\mu}^{\prime}(x)=A_{\mu}(x)+\frac{1}{g_{4}} \partial_{\mu} \hat{\Lambda}^{(+)}(x), \\
\Phi_{1}(x) \rightarrow \Phi_{1}^{\prime}(x)=e^{i g_{4} \hat{\Lambda}^{(+)}} \Phi_{1}(x), \quad \Phi_{2}(x) \rightarrow \Phi_{2}^{\prime}(x)=e^{i g_{4} \hat{\Lambda}^{(+)}} \Phi_{2}(x) .
\end{gathered}
$$

Thus, besides $\mathbb{Z}_{2}^{\prime} \times \mathbb{Z}_{2}$ symmetry, the above potential is invariant under $\mathrm{U}(1)^{\prime} \times \mathrm{U}(1)$. One $\mathrm{U}(1)$ has been gauged while the other is a remnant of the global unbroken symmetry associated with the odd gauge transformation $\left(\Lambda^{(-)}\right)$defined in eqs. (A.7)-(A.8).

One can easily see from figure 8 that the scalar potential $V\left(\Phi_{1}, \Phi_{2}\right)$ has four degenerate global minima at $\left( \pm v_{1}, 0\right)$ and $\left(0, \pm v_{2}\right)$. One can choose any of these global vacua. We 


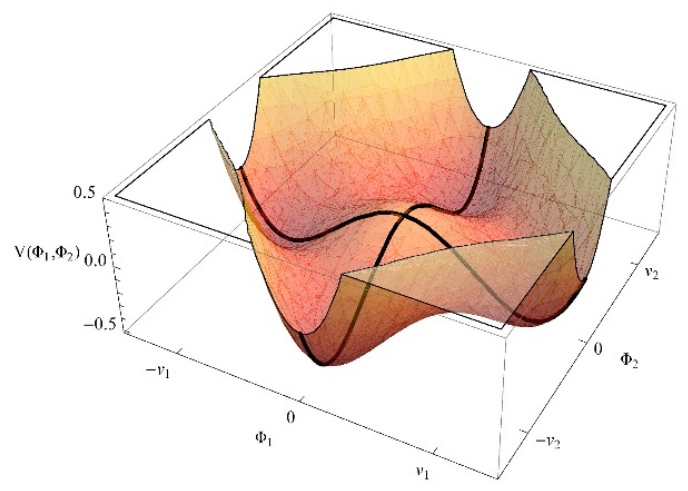

Figure 8. This graph illustrates shape of the scalar potential $V\left(\Phi_{1}, \Phi_{2}\right)$ as a function of the fields $\Phi_{1}$ and $\Phi_{2}$.

select the vacuum such that the even parity Higgs $\Phi_{1}$ acquires a vev, whereas the odd parity Higgs $\Phi_{2}$ does not. That choice of vacuum implies values of $v_{1}$ and $v_{2}$ given by,

$$
v_{1}=\frac{\mu}{\sqrt{\lambda}}, \quad v_{2}=0 .
$$

Now let us consider the fluctuations of the above fields around our choice of the vacuum,

$$
\Phi_{1}(x)=\frac{1}{\sqrt{2}}\left(v_{1}+h\right) e^{i g_{4} \pi(x)}, \quad \Phi_{2}(x)=\frac{1}{\sqrt{2}} \chi e^{i g_{4} \pi(x)} .
$$

We rewrite our effective action (A.29) only up to the quadratic order in fluctuations as

$S_{A b}^{(2)}=-\int d^{4} x\left\{\frac{1}{4} F_{\mu \nu} F^{\mu \nu}+\frac{g_{4}^{2} v_{1}^{2}}{2}\left(A_{\mu}-\partial_{\mu} \pi\right)^{2}+\frac{1}{2} \partial_{\mu} h \partial^{\mu} h+\frac{1}{2} m_{h}^{2} h^{2}+\frac{1}{2} \partial_{\mu} \chi \partial^{\mu} \chi+\frac{1}{2} m_{\chi}^{2} \chi^{2}\right\}$.

The mixing between $A_{\mu}$ and $\pi$ in the above action can be removed by an appropriate 4D gauge choice. Here we will choose the $4 \mathrm{D}$ unitary gauge such that $\pi=0$ and the gauge field acquires mass. The remaining scalars are $h$ and $\chi$ with masses

$$
m_{h}^{2}=m_{\chi}^{2}=2 \mu^{2} .
$$

Hence, the full effective Abelian action can be written in the 4D unitary gauge as

$$
\begin{aligned}
S_{A b}^{\mathrm{eff}}=-\int d^{4} x & \left\{\frac{1}{4} F_{\mu \nu} F^{\mu \nu}+\frac{1}{2} m_{A}^{2} A_{\mu} A^{\mu}+\frac{1}{2} \partial_{\mu} h \partial^{\mu} h+\frac{1}{2} m_{h}^{2} h^{2}+\frac{1}{2} \partial_{\mu} \chi \partial^{\mu} \chi+\frac{1}{2} m_{\chi}^{2} \chi^{2}\right. \\
& +\lambda v_{1} h\left(h^{2}+3 \chi^{2}\right)+\frac{1}{4} \lambda h^{4}+\frac{1}{4} \lambda \chi^{4}+\frac{3}{2} \lambda h^{2} \chi^{2} \\
& \left.+g_{4}^{2} v_{1} h A_{\mu} A^{\mu}+\frac{1}{2} g_{4}^{2}\left(h^{2}+\chi^{2}\right) A_{\mu} A^{\mu}\right\}
\end{aligned}
$$

where

$$
m_{A}^{2} \equiv g_{4}^{2} v_{1}^{2}=g_{4}^{2} \frac{\mu^{2}}{\lambda} .
$$

To summarize, the zero-mode effective theory for the Abelian case has two real scalars with equal mass and a massive gauge boson. Also, the above action is invariant under the $\mathbb{Z}_{2}$ symmetry $h \rightarrow h$ and $\chi \rightarrow-\chi$. 


\section{A.2 SSB by a vacuum expectation value of the 5D Higgs field}

In this subsection, we write the complex scalar fields $\Phi^{( \pm)}$as

$$
\left(\begin{array}{c}
\Phi^{(+)}(x, y) \\
\Phi^{(-)}(x, y)
\end{array}\right)=\frac{1}{\sqrt{2}} e^{i g_{5}\left(\pi^{(+)}(x, y) \mathbb{1}+\pi^{(-)}(x, y) \tau_{1}\right)}\left(\begin{array}{c}
v(y)+h^{(+)}(x, y) \\
h^{(-)}(x, y)
\end{array}\right) .
$$

As mentioned above, the vev $v(y)$ is only associated with the even Higgs field $\Phi^{(+)}$and it is even under the geometric $\mathbb{Z}_{2}$ parity. ${ }^{10}$ The fluctuations $h^{(+)}(x, y)$ and $\pi^{(+)}(x, y)$ are even, whereas the fluctuations $h^{(-)}(x, y)$ and $\pi^{(-)}(x, y)$ are odd under the $\mathbb{Z}_{2}$ geometric parity.

We can write the action eq. (A.9) up to quadratic order in fields as

$$
\begin{aligned}
S_{A b}^{(2)}= & -\int d^{5} x\left\{\frac{1}{4} F_{\mu \nu}^{(+)} F^{(+) \mu \nu}+\frac{1}{2} e^{2 A(y)}\left(\left(\partial_{\mu} A_{5}^{(+)}\right)^{2}+\left(\partial_{5} A_{\mu}^{(+)}\right)^{2}+g_{5}^{2} v^{2} A_{\mu}^{(+)} A^{(+) \mu}\right)\right. \\
& +\left(e^{2 A(y)} g_{5}^{2} v^{2} \pi^{(+)}-\partial_{5}\left(e^{2 A(y)} A_{5}^{(-)}\right)\right) \partial_{\mu} A^{(+) \mu}+\frac{1}{2} e^{2 A(y)}\left(\left(\partial_{\mu} h^{(+)}\right)^{2}+g_{5}^{2} v^{2}\left(\partial_{\mu} \pi^{(+)}\right)^{2}\right) \\
& +\frac{1}{2} e^{4 A(y)}\left(\left(\partial_{5} v+\partial_{5} h^{(+)}\right)^{2}+g_{5}^{2} v^{2}\left(A_{5}^{(-)}-\partial_{5} \pi^{(+)}\right)^{2}+\mu_{B}^{2}\left(v+h^{(+)}\right)^{2}\right) \\
& +\frac{1}{4} F_{\mu \nu}^{(-)} F^{(-) \mu \nu}+\frac{1}{2} e^{2 A(y)}\left(\left(\partial_{\mu} A_{5}^{(-)}\right)^{2}+\left(\partial_{5} A_{\mu}^{(-)}\right)^{2}+g_{5}^{2} v^{2} A_{\mu}^{(-)} A^{(-) \mu}\right) \\
& +\left(e^{2 A(y)} g_{5}^{2} v^{2} \pi^{(-)}-\partial_{5}\left(e^{2 A(y)} A_{5}^{(+)}\right)\right) \partial_{\mu} A^{(-) \mu}+\frac{1}{2} e^{2 A(y)}\left(\left(\partial_{\mu} h^{(-)}\right)^{2}+g_{5}^{2} v^{2}\left(\partial_{\mu} \pi^{(-)}\right)^{2}\right) \\
& \left.+\frac{1}{2} e^{4 A(y)}\left(\left(\partial_{5} h^{(-)}\right)^{2}+g_{5}^{2} v^{2}\left(A_{5}^{(+)}-\partial_{5} \pi^{(-)}\right)^{2}+\mu_{B}^{2} h^{(-) 2}\right)\right\} .
\end{aligned}
$$

where the indices are raised and lowered by the Minkowski metric. The bulk equation of motion for the background Higgs vev corresponding to the above action is

$$
\begin{aligned}
& {\left[-\partial_{5}\left(e^{4 A(y)} \partial_{5}\right)+\mu_{B}^{2} e^{4 A(y)}\right] v(y)} \\
& =-e^{4 A(y)}\left[\frac{\partial V_{\mathrm{IR}}(v)}{\partial v} \delta(y+L)+\frac{\partial V_{\mathrm{UV}}(v)}{\partial v} \delta(y)+\frac{\partial V_{\mathrm{IR}}(v)}{\partial v} \delta(y-L)\right],
\end{aligned}
$$

and the bulk equations of motion for all the fluctuations are

$$
\begin{aligned}
& {\left[-e^{2 A(y)} \square^{(4)}-\partial_{5}\left(e^{4 A(y)} \partial_{5}\right)+\mu_{B}^{2} e^{4 A(y)}\right] h^{( \pm)}(x, y)} \\
& =-e^{4 A(y)}\left[\frac{\partial^{2} V_{\mathrm{UV}}(v)}{\partial v^{2}} h^{( \pm)} \delta(y)+\frac{\partial^{2} V_{\mathrm{IR}}(v)}{\partial v^{2}} h^{( \pm)}(\delta(y-L)+\delta(y+L))\right], \\
& \square^{(4)} A_{\mu}^{( \pm)}+\partial_{5}\left(M_{A}^{2} \partial_{5} A_{\mu}^{( \pm)}\right)-M_{A}^{2} A_{\mu}^{( \pm)} \\
& =\partial_{\mu}\left(\partial^{\nu} A_{\nu}^{( \pm)}+\partial_{5}\left(e^{2 A(y)} A_{5}^{(\mp)}\right)-M_{A}^{2} \pi^{( \pm)}\right), \\
& \square^{(4)} A_{5}^{( \pm)}-\partial_{5}\left(\partial^{\nu} A_{\nu}^{(\mp)}\right)-M_{A}^{2}\left(A_{5}^{( \pm)}-\partial_{5} \pi^{(\mp)}\right)=0,
\end{aligned}
$$

\footnotetext{
${ }^{10}$ There exists also an odd parity vacuum solution but as our geometric setup is symmetric, therefore we choose the even vacuum solution $v(y)$ for the scalar field. With this choice of the background solution, the fluctuations will have a definite parity. Note that the choice of odd vacuum solution could lead to the breaking of geometric $\mathbb{Z}_{2}$ symmetry.
} 


$$
\square^{(4)} \pi^{( \pm)}-\partial^{\nu} A_{\nu}^{( \pm)}-M_{A}^{-2} \partial_{5}\left(M_{A}^{2} e^{2 A(y)}\left(A_{5}^{(\mp)}-\partial_{5} \pi^{( \pm)}\right)\right)=0,
$$

where $M_{A}^{2} \equiv g_{5}^{2} v^{2}(y) e^{2 A(y)}$. The jump conditions at the UV-brane following from the equations of motion above are:

$$
\left.\left(\partial_{5}-\frac{\partial V_{\mathrm{UV}}(v)}{\partial v}\right) v(y)\right|_{0^{+}}=0,\left.\quad\left(\partial_{5}-\frac{\partial^{2} V_{\mathrm{UV}}(v)}{\partial v^{2}}\right) h^{(+)}(x, y)\right|_{0^{+}}=0,
$$

whereas the odd fields must vanish at $y=0$. In addition, we choose the boundary conditions at $\pm L$ :

$$
\begin{aligned}
& \left.\left( \pm \partial_{5}+\frac{\partial V_{\mathrm{IR}}(v)}{\partial v}\right) v(y)\right|_{ \pm L^{\mp}}=0,\left.\quad\left( \pm \partial_{5}+\frac{\partial^{2} V_{\mathrm{IR}}(v)}{\partial v^{2}}\right) h^{( \pm)}(x, y)\right|_{ \pm L^{\mp}}=0, \\
& \partial_{\mu} A_{5}^{( \pm)}(x, y)-\left.\partial_{5} A_{\mu}^{(\mp)}(x, y)\right|_{ \pm L^{\mp}}=0, \quad A_{5}^{(\mp)}(x, y)-\left.\partial_{5} \pi^{( \pm)}(x, y)\right|_{ \pm L^{\mp}}=0,
\end{aligned}
$$

where $L^{ \pm} \equiv L \pm \epsilon$ for $\epsilon \rightarrow 0$.

We find the following vacuum solutions for $v(y)$ :

$$
v(y)=C_{1} e^{(2+\beta) k|y|}+C_{2} e^{(2-\beta) k|y|}, \quad-L \leq y \leq L,
$$

where $C_{1}$ and $C_{2}$ are the integration constants. We apply the jump condition (A.45) at $y=0$ and the boundary condition at $y= \pm L$ (A.46) to fix the two integration constants as,

$$
\begin{aligned}
C_{2} & =-\frac{\delta_{\mathrm{UV}}}{\delta_{\mathrm{UV}}+4 \beta} C_{1}, \\
C_{1} & =\sqrt{\frac{k^{3}}{\lambda_{\mathrm{IR}}}\left(\delta_{\mathrm{IR}}-\frac{\delta_{\mathrm{UV}}\left(\delta_{\mathrm{IR}}+4 \beta\right)}{\delta_{\mathrm{UV}}+4 \beta} e^{-2 \beta k L}\right)} e^{-(2+\beta) k L}\left(1-\frac{e^{-2 \beta k L} \delta_{\mathrm{UV}}}{\delta_{\mathrm{UV}}+4 \beta}\right)^{-3 / 2},
\end{aligned}
$$

where $\delta_{\mathrm{UV}} \equiv m_{\mathrm{UV}}^{2} / k^{2}-2(2+\beta)$ and $\delta_{\mathrm{IR}}$ is defined in eq. (A.26). For $k L \gg 1$ and $\beta>0$, the terms proportional to $e^{-2 \beta k L}$ are negligible in the region of interest (near the IR-brane). Hence the vacuum solution for the scalar field can be written as:

$$
v(y) \simeq \sqrt{\frac{k^{3} \delta_{\mathrm{IR}}}{\lambda_{\mathrm{IR}}}} e^{(2+\beta) k(|y|-L)} \equiv v_{4} f_{v}(y),
$$

where the constant vev $v_{4}$ and the $y$-dependent vev profile $f_{v}(y)$ are:

$$
v_{4} \equiv \sqrt{\frac{m_{K K}^{2} \delta_{\mathrm{IR}}}{\lambda_{\mathrm{IR}}(1+\beta)}}, \quad f_{v}(y) \equiv \sqrt{k(1+\beta)} e^{k L} e^{(2+\beta) k(|y|-L)} .
$$

The $y$-dependent vev profile satisfies the orthonormality condition

$$
\int_{-L}^{L} d y e^{2 A(y)} f_{v}^{2}(y)=1
$$

From the above solution we conclude that for $\lambda_{\mathrm{IR}}>0$ (as required by the positivity of the tree-level potential) one needs $\delta_{\mathrm{IR}}>0$, i.e. $m_{\mathrm{IR}}^{2} / k^{2}>2(2+\beta)$. It is worth mentioning here that the quartic term in the IR-brane potential is crucial for a non-trivial vev profile 
$v^{( \pm)}(y)$. If the quartic term would have been absent in the $V_{\mathrm{IR}}$, i.e. $\lambda_{\mathrm{IR}}=0$, then the b.c. (A.45) and (A.46) would have implied $v(y)=0$. Even though the quartic term is not in the bulk (only localized at the IR-brane), nevertheless, the b.c. imply the non-zero profile in the bulk.

In the above action there are mixing terms of $\partial_{\mu} A^{( \pm) \mu}$ with the scalars $\pi^{( \pm)}$and $A_{5}^{( \pm)}$, which can be canceled by adding the following gauge fixing Lagrangian to the above action,

$$
\begin{aligned}
S_{G F}=-\int d^{5} x & \left\{\frac{1}{2 \xi}\left[\partial_{\mu} A^{\mu(+)}-\xi\left(M_{A}^{2} \pi^{(+)}-\partial_{5}\left(e^{2 A(y)} A_{5}^{(-)}\right)\right)\right]^{2}\right. \\
& \left.+\frac{1}{2 \xi}\left[\partial_{\mu} A^{\mu(-)}-\xi\left(M_{A}^{2} \pi^{(-)}-\partial_{5}\left(e^{2 A(y)} A_{5}^{(+)}\right)\right)\right]^{2}\right\} .
\end{aligned}
$$

One can identify the Goldstone modes from the above two eqs. (A.39) and (A.54):

$$
\Pi^{( \pm)}(x, y) \equiv M_{A}^{2} \pi^{( \pm)}-\partial_{5}\left(e^{2 A(y)} A_{5}^{(\mp)}\right),
$$

along with the two pseudoscalars $\mathcal{A}_{5}^{( \pm)}(x, y)$ given as

$$
\mathcal{A}_{5}^{( \pm)}(x, y) \equiv A_{5}^{( \pm)}-\partial_{5} \pi^{(\mp)} .
$$

The resulting four pseudoscalars above along with the two $h^{( \pm)}$scalar fields agrees with the naive counting before SSB of three even-parity scalars $\left(^{(+)}(x, y), \pi^{(+)}(x, y)\right.$ and $\left.A_{5}^{(+)}(x, y)\right)$ and three odd-parity scalars $\left(h^{(-)}(x, y), \pi^{(-)}(x, y)\right.$ and $\left.A_{5}^{(-)}(x, y)\right)$. It is seen from the eq. (A.39) quadratic action that both the even and odd gauge bosons $A_{\mu}^{( \pm)}(x, y)$ acquire mass from the Higgs mechanism, whereby the two Goldstone bosons are eaten up by these gauge bosons.

In order to obtain an effective 4D Lagrangian we need to integrate the above quadratic Lagrangian over the $y$-coordinate. The first step to achieve this is to decompose all the fields in KK-modes. We will use the following decomposition,

$$
\begin{array}{llrl}
A_{\mu}^{( \pm)}(x, y) & =\sum_{n} A_{\mu n}^{( \pm)}(x) \tilde{a}_{n}^{( \pm)}(y), & & h^{( \pm)}(x, y)=\sum_{n} h_{n}^{( \pm)} \tilde{f}_{n}^{( \pm)}(y), \\
\Pi^{( \pm)}(x, y)=\sum_{n} \Pi_{n}^{( \pm)}(x) \tilde{a}_{n}^{( \pm)}(y) \tilde{m}_{A_{n}}^{( \pm)}, & \mathcal{A}_{5}^{( \pm)}(x, y)=\sum_{n} \mathcal{A}_{n}^{( \pm)}(x) \eta_{n}^{( \pm)}(y),
\end{array}
$$

where $\tilde{a}_{n}^{( \pm)}(y), \eta_{n}^{( \pm)}(y)$ and $\tilde{f}_{n}^{( \pm)}(y)$ are the $5 \mathrm{D}$ profiles for the vector fields (the same for the Goldstone fields), the pseudoscalars and the Higgs bosons, respectively. The e.o.m. for the wave-functions $\tilde{f}_{n}^{( \pm)}(y), \tilde{a}_{n}^{( \pm)}(y)$ and $\eta_{n}^{( \pm)}(y)$ are

$$
\begin{aligned}
-\partial_{5}\left(e^{4 A(y)} \partial_{5} \tilde{f}_{n}^{( \pm)}(y)\right)+\mu_{B}^{2} e^{4 A(y)} \tilde{f}_{n}^{( \pm)}(y) & =\tilde{m}_{n}^{( \pm) 2} e^{2 A(y)} \tilde{f}_{n}^{( \pm)}(y), \\
-\partial_{5}\left(e^{2 A(y)} \partial_{5} \tilde{a}_{n}^{( \pm)}(y)\right)+M_{A}^{2} \tilde{a}_{n}^{( \pm)}(y) & =\tilde{m}_{A_{n}}^{( \pm) 2} \tilde{a}_{n}^{( \pm)}(y), \\
-\partial_{5}\left(M_{A}^{-2} \partial_{5}\left(M_{A}^{2} e^{2 A(y)} \eta_{n}^{( \pm)}(y)\right)\right)+M_{A}^{2} \eta_{n}^{( \pm)}(y) & =m_{\mathcal{A}_{n}}^{( \pm) 2} \eta_{n}^{( \pm)}(y),
\end{aligned}
$$

where $\tilde{m}_{n}^{( \pm)}, \tilde{m}_{A_{n}}^{( \pm)}$and $m_{\mathcal{A}_{n}}^{( \pm)}$are the KK-masses for $h_{n}^{( \pm)}, A_{\mu n}^{( \pm)}(x)$ and $\phi_{n}^{( \pm)}(x)$. The normalization conditions for the wave-functions $\tilde{f}_{n}^{( \pm)}(y), \tilde{a}_{n}(y)$ and $\eta_{n}(y)$ are

$$
\int_{-L}^{+L} d y e^{2 A(y)} \tilde{f}_{m}^{( \pm)}(y) \tilde{f}_{n}^{( \pm)}(y)=\delta_{m n}, \quad \int_{-L}^{+L} d y \tilde{a}_{m}^{( \pm)}(y) \tilde{a}_{n}^{( \pm)}(y)=\delta_{m n}
$$




$$
\int_{-L}^{+L} d y \frac{M_{A}^{2} e^{2 A(y)}}{m_{\mathcal{A}_{m}}^{( \pm)} m_{\mathcal{A}_{n}}^{( \pm)}} \eta_{m}^{( \pm)}(y) \eta_{n}^{( \pm)}(y)=\delta_{m n}
$$

Following the general strategy mentioned in section 2.3 , we choose the $y=0$ b.c. for the even wave functions as Neumann (or mixed) b.c., whereas all the odd-mode wave functions satisfy Dirichlet b.c. at $y=0$ :

$$
\begin{aligned}
\left.\left(\partial_{5}-\frac{m_{\mathrm{UV}}^{2}}{2 k}\right) \tilde{f}_{n}^{(+)}(y)\right|_{0}=0, & \left.\tilde{f}_{n}^{(-)}(y)\right|_{0}=0, \\
\left.\partial_{5} \tilde{a}_{n}^{(+)}(y)\right|_{0}=0, & \left.\tilde{a}_{n}^{(-)}(y)\right|_{0}=0,\left.\quad \partial_{5} \eta_{n}^{(+)}(y)\right|_{0}=0,\left.\quad \eta_{n}^{(-)}(y)\right|_{0}=0 .
\end{aligned}
$$

The b.c. for wave-functions $\tilde{f}_{n}^{( \pm)}, \tilde{a}_{n}^{( \pm)}$and $\eta_{n}^{( \pm)}$at $y= \pm L$ follow from eqs. (A.46)-(A.47),

$$
\left.\left( \pm \partial_{5}-\frac{m_{\mathrm{IR}}^{2}}{2 k}+\frac{3 \lambda_{\mathrm{IR}}}{2 k^{2}} v^{2}(y)\right) \tilde{f}_{n}^{( \pm)}(y)\right|_{ \pm L^{\mp}}=0,\left.\quad \partial_{5} \tilde{a}_{n}^{( \pm)}(y)\right|_{ \pm L}=0,\left.\quad \eta_{n}^{( \pm)}(y)\right|_{ \pm L}=0 .
$$

One can also easily find the KK-decomposition of the fluctuation fields $A_{5}^{( \pm)}(x, y)$ and $\pi^{( \pm)}(x, y)$ in terms of Goldstone bosons $\Pi^{( \pm)}$and the physical scalars $\mathcal{A}_{5}^{( \pm)}$from eqs. (A.55)(A.56):

$$
\begin{aligned}
& A_{5}^{( \pm)}(x, y)=\sum_{n}\left(\frac{\Pi_{n}^{(\mp)}(x)}{\tilde{m}_{A_{n}}^{(\mp)}} \partial_{5} \tilde{a}_{n}^{(\mp)}(y)-\frac{M_{A}^{2}}{\left(m_{\mathcal{A}_{n}}^{( \pm)}\right)^{2}} \mathcal{A}_{n}^{( \pm)}(x) \eta_{n}^{( \pm)}(y)\right) \\
& \pi^{( \pm)}(x, y)=\sum_{n}\left(\frac{\Pi_{n}^{( \pm)}(x)}{\tilde{m}_{A_{n}}^{( \pm)}} \tilde{a}_{n}^{( \pm)}(y)-\frac{M_{A}^{-2}}{\left(m_{\mathcal{A}_{n}}^{(\mp)}\right)^{2}} \partial_{5}\left(M_{A}^{2} e^{2 A(y)} \eta_{n}^{(\mp)}(y)\right) \mathcal{A}_{n}^{(\mp)}(x)\right)
\end{aligned}
$$

Now we consider the low-energy effective theory obtained by assuming that the KKmass scale is high enough that we can integrate out all the heavier KK modes and keep only the zero-modes of the theory. From here on, we choose the unitary gauge such that $\xi \rightarrow \infty$ which implies $\Pi_{n}^{( \pm)}(x) \rightarrow 0$. Moreover, with our choice of boundary conditions for $a_{0}^{(-)}(y)$ and $\eta_{0}^{( \pm)}(y)$ in eqs. (A.65) and (A.66) one can see that the corresponding wave-functions for zero-modes are vanishing, i.e. there will be no zero-modes $A_{0 \mu}^{(-)}(x)$ and $\mathcal{A}_{0}^{( \pm)}(x)$ in our effective theory. The $y$-dependent vev and the zero-mode profiles for even and odd Higgs are given by

$$
\begin{gathered}
f_{v}(y) \equiv \sqrt{k(1+\beta)} e^{k L} e^{(2+\beta) k(|y|-L)}, \\
\tilde{f}_{0}^{( \pm)}(|y|) \approx \sqrt{k(1+\beta)} e^{k L} e^{(2+\beta) k(|y|-L)}, \quad \tilde{f}_{0}^{(-)}(y)=\epsilon(y) \tilde{f}_{0}^{(-)}(|y|),
\end{gathered}
$$

where $\mu^{2} \equiv(1+\beta) m_{K K}^{2} \delta_{\mathrm{IR}}$ and $\lambda \equiv \lambda_{\mathrm{IR}}(1+\beta)^{2}$. It is important to comment here that at the leading order the vev profile and zero-mode profiles are the same. However, there are finite corrections which are suppressed by $\mathcal{O}\left(m_{h}^{2} / m_{K K}^{2}\right)$ as given below

$$
\frac{\tilde{f}_{0}^{( \pm)}(|y|)}{f_{v}(|y|)}=1+\frac{m_{h}^{2}}{m_{K K}^{2}}\left(\frac{1-e^{2 k(|y|-L)}}{4(1+\beta)}+\mathcal{O}\left(\frac{m_{h}^{2}}{m_{K K}^{2}}\right)\right) .
$$




\begin{tabular}{|c|c|c|c|c|c|c|}
\hline & \multicolumn{3}{|c|}{ EWSB by KK mode vev } & \multicolumn{3}{|c|}{ EWSB by 5D Higgs vev } \\
\hline $5 \mathrm{D}$ fields & KK-modes & $n=0$ & $n \neq 0$ & KK-modes & $n=0$ & $n \neq 0$ \\
\hline $\operatorname{Re} \Phi^{(+)}$ & $\phi_{n}^{(+)}(x)$ & $\checkmark$ & $\checkmark$ & $h_{n}^{(+)}(x)$ & $\checkmark$ & $\checkmark$ \\
\hline $\operatorname{Re} \Phi^{(-)}$ & $\phi_{n}^{(-)}(x)$ & $\checkmark$ & $\checkmark$ & $h_{n}^{(-)}(x)$ & $\checkmark$ & $\checkmark$ \\
\hline $\operatorname{Im} \Phi^{(+)}$ & $\pi_{n}^{(+)}(x)$ & $\boldsymbol{x}(4 \mathrm{D}$ g.c. $)$ & $\checkmark$ & $\Pi_{n}^{(+)}(x)$ & $\boldsymbol{x}(4 \mathrm{D}$ g.c. $)$ & $\boldsymbol{x}(4 \mathrm{D}$ g.c. $)$ \\
\hline $\operatorname{Im} \Phi^{(-)}$ & $\pi_{n}^{(-)}(x)$ & $\boldsymbol{x}$ (b.c.) & $\checkmark$ & $\Pi_{n}^{(-)}(x)$ & $\boldsymbol{x}(4 \mathrm{D}$ g.c. $)$ & $\boldsymbol{x}(4 \mathrm{D}$ g.c. $)$ \\
\hline$A_{5}^{(+)}$ & $A_{5 n}^{(+)}(x)$ & $\boldsymbol{x}(5 \mathrm{D}$ g.c. $)$ & $\boldsymbol{x}(5 \mathrm{D}$ g.c. $)$ & $\mathcal{A}_{n}^{(+)}(x)$ & $\boldsymbol{x}$ (b.c.) & $\checkmark$ \\
\hline$A_{5}^{(-)}$ & $A_{5 n}^{(-)}(x)$ & $\boldsymbol{x}(5 \mathrm{D}$ g.c. $)$ & $\boldsymbol{x}(5 \mathrm{D}$ g.c. $)$ & $\mathcal{A}_{n}^{(-)}(x)$ & $\boldsymbol{x}$ (b.c.) & $\checkmark$ \\
\hline$A_{\mu}^{(+)}$ & $A_{\mu n}^{(+)}(x)$ & $\checkmark$ & $\checkmark$ & $A_{\mu n}^{(+)}(x)$ & $\checkmark$ & $\checkmark$ \\
\hline$A_{\mu}^{(-)}$ & $A_{\mu n}^{(-)}(x)$ & $\boldsymbol{x}$ (b.c.) & $\checkmark$ & $A_{\mu n}^{(-)}(x)$ & $\boldsymbol{x}$ (b.c.) & $\checkmark$ \\
\hline
\end{tabular}

Table 1. Comparison of dynamical d.o.f. between KK-mode-vev and 5D-Higgs-vev EWSB. The b.c. (boundary condition) and g.c. (gauge choice) show why a given mode is not present in the corresponding effective theory. Note that $\Pi_{n}^{( \pm)}$is a mixture of $\pi_{n}^{( \pm)}$and $A_{5 n}^{( \pm)}$, see eq. (A.55).

We can now write down the effective theory for the zero-modes in the unitary gauge:

$$
\begin{aligned}
S_{\text {eff }}=-\int d^{4} x & \left\{\frac{1}{4} F_{\mu \nu} F^{\mu \nu}+\frac{1}{2} \tilde{m}_{A}^{2} A_{\mu} A^{\mu}+\frac{1}{2} \partial_{\mu} h \partial^{\mu} h+\frac{1}{2} \tilde{m}_{h}^{2} h^{2}+\frac{1}{2} \partial_{\mu} \chi \partial^{\mu} \chi+\frac{1}{2} \tilde{m}_{\chi}^{2} \chi^{2}\right. \\
+ & \frac{1}{4} \lambda h^{4}+\frac{1}{4} \lambda \chi^{4}+\frac{3}{2} \lambda h^{2} \chi^{2}+\lambda v_{4} h\left(h^{2}+3 \chi^{2}\right) \\
+ & \left.\tilde{g}_{4}^{2} v_{4} h A_{\mu} A^{\mu}+\frac{1}{2} \tilde{g}_{4}^{2}\left(h^{2}+\chi^{2}\right) A_{\mu} A^{\mu}\right\}
\end{aligned}
$$

where we have denoted $A_{0 \mu}^{(+)}(x) \equiv A_{\mu}(x)$ and we have suppressed the zero-mode subscript ' 0 ' for all modes. After some algebra, using the boundary conditions, one can find the masses of the zero-mode scalars and gauge boson at the leading order:

$$
\tilde{m}_{h}^{2}=\tilde{m}_{\chi}^{2} \simeq 2 \mu^{2}, \quad \quad \tilde{m}_{A}^{2} \simeq \frac{1}{2 L} \int_{-L}^{L} d y M_{A}^{2}=\tilde{g}_{4}^{2} v_{4}^{2},
$$

where $\mu^{2} \equiv(1+\beta) m_{K K}^{2} \delta_{\mathrm{IR}}, v_{4} \equiv \mu / \lambda$ and $\tilde{g}_{4} \equiv g_{5} / \sqrt{2 L}$.

Comparison. In order to facilitate comparison between the two approaches, we collect information concerning all the low-energy degrees of freedom for both pictures in table 1 . Comparing the effective theories obtained within EWSB induced by the Higgs KK-mode vev and by a 5D-Higgs vev in (A.36) and (A.72) one finds that both approaches give exactly the same zero-mode effective theory up to $\mathcal{O}\left(m_{h}^{2} / m_{K K}^{2} \sim 10^{-3}\right)$ corrections. We have checked that the scalar masses are exactly same to all orders in the expansion parameter $m_{h}^{2} / m_{K K}^{2}$. In contrast, the gauge boson masses and the couplings can have subleading differences of order $\mathcal{O}\left(m_{h}^{2} / m_{K K}^{2}\right)$. Note that we have neglected all the effects due to the non-zero KK-modes, such effects being suppressed by their masses, i.e. $\mathcal{O}\left(m_{h}^{2} / m_{n}^{2}\right)$. Hence we conclude that the two approaches to EWSB discussed above give the same low-energy (zero-mode) effective theory aside from small deviations of order $\mathcal{O}\left(m_{h}^{2} / m_{K K}^{2}\right)$. To make 


\begin{tabular}{|l|l|c|}
\hline EWSB by KK mode vev & EWSB by 5D Higgs vev & Comment \\
\hline$f_{0}(y) \simeq \sqrt{k(1+\beta)} e^{k L} e^{(2+\beta) k(|y|-L)}$ & $\tilde{f}_{0}(y) \simeq \sqrt{k(1+\beta)} e^{k L} e^{(2+\beta) k(|y|-L)}$ & same \\
$v_{1}^{2}=\frac{\mu^{2}}{\lambda}\left(1-\mathcal{O}\left(\frac{m_{h}^{2}}{m_{K K}^{2}}\right)\right)$ & $v_{4}^{2}=\frac{\mu^{2}}{\lambda} \quad$ and $\quad f_{v}(y)=\tilde{f}_{0}(y)$ & $\mathcal{O}\left(\frac{m_{h}^{2}}{m_{K K}^{2}}\right)$ \\
$m_{h}^{2}=m_{\chi}^{2}=2 \mu^{2}\left(1-\mathcal{O}\left(\frac{m_{h}^{2}}{m_{K K}^{2}}\right)\right)$ & $\tilde{m}_{h}^{2}=\tilde{m}_{\chi}^{2}=2 \mu^{2}\left(1-\mathcal{O}\left(\frac{m_{h}^{2}}{m_{K K}^{2}}\right)\right)$ & same \\
$a_{0}(y)=\frac{1}{\sqrt{2 L}}$ & $\tilde{a}_{0}(y)=\frac{1}{\sqrt{2 L}}\left(1+\mathcal{O}\left(\frac{m_{h}^{2}}{m_{K K}^{2}}\right)\right)$ & $\mathcal{O}\left(\frac{m_{h}^{2}}{m_{K K}^{2}}\right)$ \\
$g_{4}=\frac{g_{5}}{\sqrt{2 L}}$ & $\tilde{g}_{4}=\frac{g_{5}}{\sqrt{2 L}}\left(1-\mathcal{O}\left(\frac{m_{h}^{2}}{m_{K K}^{2}}\right)\right)$ & $\mathcal{O}\left(\frac{m_{h}^{2}}{m_{K K}^{2}}\right)$ \\
$m_{A}^{2}=\frac{g_{5}^{2}}{2 L} \frac{\mu^{2}}{\lambda}\left(1-\mathcal{O}\left(\frac{m_{h}^{2}}{m_{K K}^{2}}\right)\right)$ & $\tilde{m}_{A}^{2}=\frac{g_{5}^{2}}{2 L} \frac{\mu^{2}}{\lambda}\left(1-\mathcal{O}\left(\frac{m_{h}^{2}}{m_{K K}^{2}}\right)\right)$ & $\mathcal{O}\left(\frac{m_{h}^{2}}{m_{K K}^{2}}\right)$ \\
\hline
\end{tabular}

Table 2. Comparison of the effective parameters in terms of the fundamental parameters of the $5 \mathrm{D}$ theory, where $\mu^{2} \equiv(1+\beta) m_{K K}^{2} \delta_{\mathrm{IR}}$ and $\lambda=(1+\beta)^{2} \lambda_{\mathrm{IR}}$. Here we explicitly show the presence of corrections of order of the expansion parameter, $m_{h}^{2} / m_{K K}^{2} \sim 10^{-3}$, and we neglect effects $\mathcal{O}\left(e^{-2 \beta k L}\right)$; the latter ones are extremely small for $\beta>0$ and $k L \sim 35$.

this comparison more transparent we summarize the parameters of both effective theories in terms of the fundamental parameters of the $5 \mathrm{D}$ theory in table 2 . The observed agreement at the zero-mode level of the effective theory is a non-trivial verification of the results obtained here.

Open Access. This article is distributed under the terms of the Creative Commons Attribution License (CC-BY 4.0), which permits any use, distribution and reproduction in any medium, provided the original author(s) and source are credited.

\section{References}

[1] L. Randall and R. Sundrum, A large mass hierarchy from a small extra dimension, Phys. Rev. Lett. 83 (1999) 3370 [hep-ph/9905221] [INSPIRE].

[2] L. Randall and R. Sundrum, An alternative to compactification, Phys. Rev. Lett. 83 (1999) 4690 [hep-th/9906064] [INSPIRE].

[3] W.D. Goldberger and M.B. Wise, Modulus stabilization with bulk fields, Phys. Rev. Lett. 83 (1999) 4922 [hep-ph/9907447] [INSPIRE].

[4] O. DeWolfe, D.Z. Freedman, S.S. Gubser and A. Karch, Modeling the fifth-dimension with scalars and gravity, Phys. Rev. D 62 (2000) 046008 [hep-th/9909134] [INSPIRE].

[5] H. Davoudiasl, J.L. Hewett and T.G. Rizzo, Bulk gauge fields in the Randall-Sundrum model, Phys. Lett. B 473 (2000) 43 [hep-ph/9911262] [INSPIRE].

[6] Y. Grossman and M. Neubert, Neutrino masses and mixings in nonfactorizable geometry, Phys. Lett. B 474 (2000) 361 [hep-ph/9912408] [INSPIRE].

[7] S. Chang, J. Hisano, H. Nakano, N. Okada and M. Yamaguchi, Bulk standard model in the Randall-Sundrum background, Phys. Rev. D 62 (2000) 084025 [hep-ph/9912498] [INSPIRE].

[8] T. Gherghetta and A. Pomarol, Bulk fields and supersymmetry in a slice of AdS, Nucl. Phys. B 586 (2000) 141 [hep-ph/0003129] [INSPIRE]. 
[9] S.J. Huber and Q. Shafi, Fermion masses, mixings and proton decay in a Randall-Sundrum model, Phys. Lett. B 498 (2001) 256 [hep-ph/0010195] [INSPIRE].

[10] K. Agashe, A. Delgado, M.J. May and R. Sundrum, RS1, custodial isospin and precision tests, JHEP 08 (2003) 050 [hep-ph/0308036] [INSPIRE].

[11] M.A. Luty and T. Okui, Conformal technicolor, JHEP 09 (2006) 070 [hep-ph/0409274] [INSPIRE].

[12] N. Arkani-Hamed, M. Porrati and L. Randall, Holography and phenomenology, JHEP 08 (2001) 017 [hep-th/0012148] [INSPIRE].

[13] R. Rattazzi and A. Zaffaroni, Comments on the holographic picture of the Randall-Sundrum model, JHEP 04 (2001) 021 [hep-th/0012248] [INSPIRE].

[14] H. Davoudiasl, B. Lillie and T.G. Rizzo, Off-the-wall Higgs in the universal Randall-Sundrum model, JHEP 08 (2006) 042 [hep-ph/0508279] [INSPIRE].

[15] G. Cacciapaglia, C. Csáki, G. Marandella and J. Terning, The gaugephobic Higgs, JHEP 02 (2007) 036 [hep-ph/0611358] [INSPIRE].

[16] A. Falkowski and M. Pérez-Victoria, Electroweak breaking on a soft wall, JHEP 12 (2008) 107 [arXiv:0806.1737] [inSPIRE].

[17] A. Azatov, M. Toharia and L. Zhu, Higgs mediated FCNC's in warped extra dimensions, Phys. Rev. D 80 (2009) 035016 [arXiv:0906.1990] [InSPIRE].

[18] J.A. Cabrer, G. von Gersdorff and M. Quirós, Warped electroweak breaking without custodial symmetry, Phys. Lett. B 697 (2011) 208 [arXiv:1011.2205] [INSPIRE].

[19] J.A. Cabrer, G. von Gersdorff and M. Quirós, Suppressing electroweak precision observables in 5D warped models, JHEP 05 (2011) 083 [arXiv: 1103.1388] [INSPIRE].

[20] J.A. Cabrer, G. von Gersdorff and M. Quirós, Improving naturalness in warped models with a heavy bulk Higgs boson, Phys. Rev. D 84 (2011) 035024 [arXiv:1104.3149] [INSPIRE].

[21] M. Geller, S. Bar-Shalom and A. Soni, Higgs-radion unification: radius stabilization by an SU(2) bulk doublet and the 126 GeV scalar, Phys. Rev. D 89 (2014) 095015 [arXiv: 1312.3331] [INSPIRE].

[22] P.R. Archer, The fermion mass hierarchy in models with warped extra dimensions and a bulk Higgs, JHEP 09 (2012) 095 [arXiv: 1204.4730] [INSPIRE].

[23] M. Frank, N. Pourtolami and M. Toharia, Higgs bosons in warped space, from the bulk to the brane, Phys. Rev. D 87 (2013) 096003 [arXiv:1301.7692] [INSPIRE].

[24] R. Malm, M. Neubert, K. Novotny and C. Schmell, 5D perspective on Higgs production at the boundary of a warped extra dimension, JHEP 01 (2014) 173 [arXiv:1303.5702] [INSPIRE].

[25] P. Cox, A.D. Medina, T.S. Ray and A. Spray, Radion/dilaton-Higgs mixing phenomenology in light of the LHC, JHEP 02 (2014) 032 [arXiv:1311.3663] [INSPIRE].

[26] P.R. Archer, M. Carena, A. Carmona and M. Neubert, Higgs production and decay in models of a warped extra dimension with a bulk Higgs, JHEP 01 (2015) 060 [arXiv: 1408.5406] [INSPIRE].

[27] B.M. Dillon and S.J. Huber, Non-custodial warped extra dimensions at the LHC?, JHEP 06 (2015) 066 [arXiv:1410.7345] [INSPIRE]. 
[28] K. Agashe, A. Azatov, Y. Cui, L. Randall and M. Son, Warped dipole completed, with a tower of Higgs bosons, JHEP 06 (2015) 196 [arXiv:1412.6468] [INSPIRE].

[29] A.M. Iyer, K. Sridhar and S.K. Vempati, Bulk RS models, electroweak precision tests and the $125 \mathrm{GeV}$ Higgs, arXiv: 1502.06206 [INSPIRE].

[30] A. Ahmed and B. Grzadkowski, Brane modeling in warped extra-dimension, JHEP 01 (2013) 177 [arXiv: 1210.6708] [INSPIRE].

[31] A. Ahmed, L. Dulny and B. Grzadkowski, Generalized Randall-Sundrum model with a single thick brane, Eur. Phys. J. C 74 (2014) 2862 [arXiv: 1312.3577] [INSPIRE].

[32] Planck collaboration, P.A.R. Ade et al., Planck 2015 results. XIII. Cosmological parameters, arXiv:1502.01589 [INSPIRE].

[33] G. Servant and T.M.P. Tait, Is the lightest Kaluza-Klein particle a viable dark matter candidate?, Nucl. Phys. B 650 (2003) 391 [hep-ph/0206071] [INSPIRE].

[34] H.-C. Cheng, J.L. Feng and K.T. Matchev, Kaluza-Klein dark matter, Phys. Rev. Lett. 89 (2002) 211301 [hep-ph/0207125] [INSPIRE].

[35] K. Agashe and G. Servant, Warped unification, proton stability and dark matter, Phys. Rev. Lett. 93 (2004) 231805 [hep-ph/0403143] [INSPIRE].

[36] G. Panico, E. Ponton, J. Santiago and M. Serone, Dark matter and electroweak symmetry breaking in models with warped extra dimensions, Phys. Rev. D 77 (2008) 115012 [arXiv: 0801.1645] [INSPIRE].

[37] E. Ponton and L. Randall, TeV scale singlet dark matter, JHEP 04 (2009) 080 [arXiv:0811.1029] [INSPIRE].

[38] L. Vechi, Majorana dark matter in warped extra dimensions, Phys. Rev. D 90 (2014) 025017 [arXiv: 1310.7862] [INSPIRE].

[39] T. Gherghetta and B. von Harling, A warped model of dark matter, JHEP 04 (2010) 039 [arXiv: 1002.2967] [INSPIRE].

[40] B. von Harling and K.L. McDonald, Secluded dark matter coupled to a hidden CFT, JHEP 08 (2012) 048 [arXiv: 1203.6646] [INSPIRE].

[41] A.R. Frey, R.J. Danos and J.M. Cline, Warped Kaluza-Klein dark matter, JHEP 11 (2009) 102 [arXiv:0908.1387] [INSPIRE].

[42] K. Agashe, A. Falkowski, I. Low and G. Servant, KK parity in warped extra dimension, JHEP 04 (2008) 027 [arXiv: 0712.2455] [INSPIRE].

[43] A.D. Medina and E. Ponton, Warped universal extra dimensions, JHEP 06 (2011) 009 [arXiv: 1012.5298] [INSPIRE].

[44] A.D. Medina and E. Ponton, Warped radion dark matter, JHEP 09 (2011) 016 [arXiv: 1104.4124] [INSPIRE].

[45] Z. Lalak and R. Matyszkiewicz, Boundary terms in brane worlds, JHEP 11 (2001) 027 [hep-th/0110141] [INSPIRE].

[46] C. Csáki, M.L. Graesser and G.D. Kribs, Radion dynamics and electroweak physics, Phys. Rev. D 63 (2001) 065002 [hep-th/0008151] [INSPIRE].

[47] A. Carmona and M. Chala, Composite dark sectors, JHEP 06 (2015) 105 [arXiv: 1504.00332] [INSPIRE]. 
[48] B. Grzadkowski and J. Wudka, Pragmatic approach to the little hierarchy problem: the case for dark matter and neutrino physics, Phys. Rev. Lett. 103 (2009) 091802 [arXiv: 0902.0628] [INSPIRE].

[49] C.F. Kolda and H. Murayama, The Higgs mass and new physics scales in the minimal standard model, JHEP 07 (2000) 035 [hep-ph/0003170] [INSPIRE].

[50] J.A. Casas, J.R. Espinosa and I. Hidalgo, Implications for new physics from fine-tuning arguments. 1. Application to SUSY and seesaw cases, JHEP 11 (2004) 057 [hep-ph/0410298] [INSPIRE].

[51] R. Barbieri and G.F. Giudice, Upper bounds on supersymmetric particle masses, Nucl. Phys. B 306 (1988) 63 [INSPIRE].

[52] A. Carmona, E. Ponton and J. Santiago, Phenomenology of non-custodial warped models, JHEP 10 (2011) 137 [arXiv: 1107.1500] [INSPIRE].

[53] Particle Data Group collaboration, K.A. Olive et al., Review of particle physics, Chin. Phys. C 38 (2014) 090001 [inSPIRE].

[54] E.W. Kolb and M.S. Turner, The early universe, Front. Phys. 69 (1990) 1 [INSPIRE]. 